\title{
1 Unravelling the Interactions between Surface-Active Ionic Liquids and Triblock-Copolymers for the Design of Thermal Responsive Systems
}

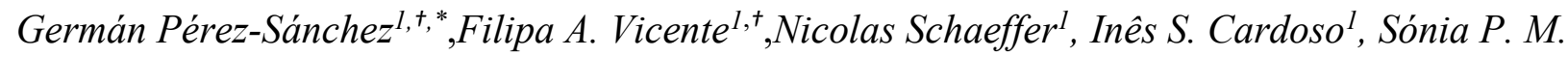
Ventura ${ }^{1}$, Miguel Jorge ${ }^{2,}{ }^{*}$ and João A. P. Coutinho ${ }^{1}$

${ }^{1}$ CICECO, Departamento de Química, Universidade de Aveiro, 3810-193 Aveiro, Portugal

${ }^{2}$ Department of Chemical and Process Engineering, University of Strathclyde, 75 Montrose Street, Glasgow G1 1XJ, United Kingdom

*Corresponding authors

${ }^{\dagger}$ Both authors contributed equally

Campus Universitário de Santiago, Universidade de Aveiro, Aveiro, Portugal

Tel: +351-234-370200; Fax: +351-234-370084; E-mail addresses: german@,ua.pt, miguel.jorge@strath.ac.uk 


\section{ABSTRACT}

2 The tuneable properties of surface-active ionic liquids (SAIL) and Pluronics are dramatically 3 magnified by combining them in aqueous solutions. The thermo-controlled character of both,

4 essential in the extraction of valuable compounds, can be fine-tuned by properly selecting the

5 Pluronic and SAIL nature. However, further understanding of the nanoscale interactions directing

6 the aggregation in these complex mixtures is needed to effectively design and control these

7 systems. In this work, a simple and transferable coarse-grained model for molecular dynamics

8 simulations, based on the MARTINI force field, is presented to study the impact of SAILs in

9 Pluronics aggregation in aqueous solutions. The diverse amphiphilic characteristics and micelle

10 morphologies were exemplified by selecting four archetypical non-ionic Pluronics; two normal,

11 L-31 and L-35 and, two reverse, 10R5 and 31R1. The impact of the alkyl-chain length and the

12 headgroup nature were evaluated with the imidazolium $\left[\mathrm{C}_{10 \mathrm{mim}}\right] \mathrm{Cl},\left[\mathrm{C}_{14 \mathrm{mim}}\right] \mathrm{Cl}$ and

13 phosphonium-based $\left[\mathrm{P}_{4,4,4,14}\right] \mathrm{Cl}$ SAILs. Cloud point temperature (CPT) measurements at different

14 Pluronic concentrations with $0.3 \%$ wt of SAIL in aqueous solution emphasised the distinct impact

15 of SAIL nature on the thermo-response behaviour.

16 The main effect of SAIL addition to non-ionic Pluronics aqueous solutions is the formation of

17 Pluronic/SAIL hybrid micelles, where the presence of SAIL molecules introduces a charged

18 character to the micelle surface. Thus, additional energy is necessary to induce micelle

19 aggregation, leading to the observed increase in the experimental CPT curves. The SAIL showed

20 a relatively weak impact in Pluronic micelles with relatively high PPG hydrophobic content,

21 whereas this effect was more evident when the Pluronic hydrophobic/hydrophilic strength is

22 balanced. A detailed analysis of the Pluronic/SAIL micelle density profiles showed that the

23 phosphonium head groups were positioned inside the micelle core, whereas smaller imidazolium

24 head groups were placed much closer to the hydrophilic PEG corona, leading to a distinct effect

25 on the cloud point temperature for those two classes of SAIL. Herein, the phosphonium-based

26 SAIL induces a lower repulsion between neighbouring micelles than the imidazolium-based

27 SAILs, resulting in a less pronounced increase of the CPT. The model presented in here offers, for

28 the first time, an intuitive and powerful tool to unravel the complex thermo-response behaviour of

29 Pluronic and SAIL mixtures and support the design of tailor-made thermal controlled solvents. 
1 Keywords: coarse-grain computer model, molecular dynamic simulations, micellar self-assembly,

2 Pluronics and ionic liquid mixtures 


\section{Introduction}

2 Triblock copolymers, also known as Pluronics or Poloxamers, are emerging as promising biocompatible candidates for the development of tuneable and integrated purification platforms as evidenced by their increasing application in the recovery of value-added compounds such as 5 pharmaceuticals, ${ }^{1}$ biomolecules ${ }^{2}$ and metals ${ }^{3}$ amongst others. Pluronics are thermo-responsive copolymers constituted by units of poly(oxyethylene)-poly(oxypropylene)-poly(oxyethylene) $(\mathrm{EO})_{\mathrm{n}}(\mathrm{PO})_{\mathrm{m}}(\mathrm{EO})_{\mathrm{n}}{ }^{4} \quad$ or poly(oxypropylene-poly(oxyethylene)- poly(oxypropylene -

$8 \quad(\mathrm{PO})_{\mathrm{m}}(\mathrm{EO})_{\mathrm{n}}(\mathrm{PO})_{\mathrm{m}}{ }^{5}$. They present well-known lower critical solution temperature (LCST) behaviour, allowing at the reversible formation of aqueous micellar two-phase systems (AMTPS) by temperature adjustment, simplifying the process development and decreasing the necessity of

11 large quantities of a tertiary compound to promote phase separation. The presence of both EO and 12 PO units allows to fine tune the amphiphilic properties of Pluronics. ${ }^{6}$ The latter aggregate in 13 aqueous solutions to form micelles constituted by a polypropylene glycol (PPG) hydrophobic core 14 with the polyethylene glycol (PEG) segments arranged outwards to form the hydrophilic corona. 15 Upon temperature increase, the PPG hydrophobic effect becomes stronger while the PEG segments 16 dehydrate due to conformational changes in the EO groups, resulting in a two-phase separation at 17 a temperature known as the cloud point. ${ }^{6}$ The versatility of Pluronics as solvents stems from this 18 self-organisation, which is influenced by the ratio of PO to EO units, the PO to EO sequence 19 (normal and reverse Pluronics), and the molecular weight. ${ }^{7}$

20 Through judicious adjustment of the biphasic environment, conditions promoting the selective 21 partition of target compounds versus contaminants can be achieved. One method to enhance 22 selectivity is through the addition of affinity ligands that significantly partition to one phase, 23 thereby modifying its properties and extraction characteristics. ${ }^{8}$ Ionic liquids (ILs) are promising 24 candidates as affinity ligands due to their ability to solvate a wide range of compounds (polar and apolar) and to act either as a solvent or a catalyst. Surface active ILs (SAILs) are a subset of ILs, with either the cation or anion possessing an alkyl chain longer than six carbon atoms allowing it 27 to self-aggregate in aqueous solutions. ${ }^{9}$ The incorporation of small quantities of SAILs into micellar systems overcomes the major issues traditionally associated with ILs, namely their cost and viscosity. Furthermore, Dong et al. ${ }^{10}$ showed that imidazolium-based SAILs can have a better

30 performance than conventional cationic surfactants with lower concentrations required to promote 31 a similar effect. However, studies on the incorporation of SAILs into mixed micelles with both 
1 ionic and non-ionic surfactants reveal the marked variability in the resulting properties of the 2 system compared to its pure components, including its cloud-point., ${ }^{91,20,12-19}$ A deeper 3 understanding of the molecular-scale interactions controlling these complex systems often remains 4 lacking.

5 In spite of the individual advantages of Pluronics and SAILs, only a few reports are available on the effect of ILs on Pluronics, either with short or long alkyl side chain ILs. They are mainly focused on the influence of SAILs in small concentration ranges, focusing on the critical micelle 8 concentration (CMC), the critical micellization temperature (CMT) and/or the micelle size. Higher concentrations required for extraction purposes are often not considered in these studies despite the pronounced effect of concentration and nature of each compound on the phase behaviour, 11 namely on the formation of micelles and long range ordered structures, which have different thermal responses. A detailed knowledge of the molecular scale interactions driving the self13 assembly of these mixtures is of great importance to design and expand the range of thermal 14 responsive copolymer-SAILs systems. In this respect, computer simulations can rationalize 15 available experimental data and provide a platform for the inexpensive screening of potential 16 systems. Our group recently developed a coarse-grain (CG) model for molecular dynamics (MD) 17 computer simulations of Pluronic aqueous solutions. ${ }^{7}$ The model captured the complexities of 18 Pluronic micelle formation and the influence of the PPG content, the PEG to PPG ratio or the 19 Pluronic molecular weight on the cloud point temperature. As such, it provides a valuable 20 framework to study the influence of SAIL addition to Pluronic aqueous solutions on the thermal 21 response of the system and complement existing experimental studies. ${ }^{18,19,28,29,20-27}$

22 In this work four selected Pluronics were studied in conjunction with imidazolium and 23 phosphonium-based SAILs to determine the influence of the ILs as additives on the cloud points 24 and thermo-responsiveness of the system. Both normal (PEG-PPG-PEG) and reverse (PPG-PEG25 PPG) Pluronics were studied as these structural changes induce major micelle surface variations 26 from star-like to flower-like shaped micelles, respectively, as well as the occurrence of physical 27 cross linkage between reverse Pluronic micelles. ${ }^{7}$ Furthermore, the effect of the polymer molecular 28 weight and PPG to PEG ratio in mixed systems was also assessed. Finally, the influence of the 29 SAILs cationic alkyl chain length and the nature of the cation, namely imidazolium or quaternary 30 phosphonium, on the thermo-responsive properties of the system was determined. In doing so, the 31 conclusions obtained herein can be extended to the application, understanding and design of 
1 Pluronic-based responsive formulations of relevance for the development of sustainable and 2 integrated separation systems.

6 All studied triblock copolymers studied in this work were purchased from Sigma-Aldrich, 7 namely Pluronic L-31, L-35, 31R1 and 10R5 (see Table S1). Both the imidazolium: 1-decyl-38 methylimidazolium chloride - [ $\left.\mathrm{C}_{10} \mathrm{mim}\right] \mathrm{Cl}$ (> $98 \mathrm{wt} \%$ ), 1-dodecyl-3-methylimidazolium chloride $9-\left[\mathrm{C}_{12} \mathrm{mim}\right] \mathrm{Cl}(>98 \mathrm{wt} \%)$ and 1-methyl-3-tetradecylimidazolium chloride $-\left[\mathrm{C}_{14} \mathrm{mim}\right] \mathrm{Cl}$ (> 98 $10 \mathrm{wt} \%$ ), and the phosphonium-based SAIL, represented by tributyltetradecylphosphonium chloride 11 - $\left[\mathrm{P}_{4,4,4,14]} \mathrm{Cl}\right.$ (95\%), were acquired at Iolitec (Ionic Liquid Technologies, Heilbronn, Germany).

12 McIlvaine buffer $(0.18 \mathrm{M})$ at $\mathrm{pH} 7.0$ was used as solvent, being composed of sodium phosphate 13 dibasic heptahydrate (purity $\geq 99 \%$ ) and citric acid monohydrate (purity $\geq 99 \%$ ), which were 14 obtained from Panreac AppliChem.

15 The cloud point determination was established in Mcllvaine buffer solutions, since this is the medium of preference to work with $\mathrm{pH}$ sensitive or labile biomolecules, and at copolymer 17 concentrations ranging from 0.5 to $17.5 \mathrm{wt} \%$ and SAIL concentrations of $0.3 \mathrm{wt} \%$. For each system, three replicas were determined, and the average value calculated. The cloud points were measured through the visual determination of the onset turbidity of a solution heated in a temperature controlled water bath with an error of $\pm 0.01^{\circ} \mathrm{C}$ following a methodology described elsewhere. ${ }^{30,31}$ The full results are shown in Figure S1.

\subsection{Simulation Details}

24 The CG models for Pluronics, ${ }^{7}$ phosphonium ${ }^{32}$ and imidazolium ${ }^{33}$ ILs used in this work were successfully developed, validated and discussed in recent publications by the authors, and are shown in Figure 1. They are based on the MARTINI framework, ${ }^{34}$ with appropriate adaptations

27 for the specific systems studied here. The applicability and transferability of the MARTINI CG approach has been widely documented in the recent literature, ${ }^{35-37}$ and the reader is referred to those studies for additional details. Regarding the phosphonium CG model, the regular MARTINI

30 water was used bearing in mind the high computational costs of simulating systems with 31 MARTINI polarizable water, four times longer compared with regular water, ${ }^{38,39}$ and the PME to 
1 cope with the electrostatics requiring $\sim 3$ times longer than cut off treatment, ${ }^{40}$ both heavily 2 increasing the simulation time. In addition, the Pluronic aqueous solution concentration is $1 \mathrm{wt} \%$, 3 requiring a huge number of water molecules. A simulation test was carried out where two versions 4 of a $\left[\mathrm{P}_{4,4,4,14}\right] \mathrm{Cl}$ aqueous solution in the micellar regime $7 \% \mathrm{wt}$ and $298 \mathrm{~K}$ entailing 100 ILs. The 5 micellar distribution equilibrium was attained after $90 \mathrm{~ns}$ in the case of the regular MARTINI water whereas $400 \mathrm{~ns}$ was required to attain the same micellar distribution of one micelle using the polarizable water. Figure $\mathbf{S 2}$ shows the $\left[\mathrm{P}_{4,4,4,14}\right] \mathrm{Cl}$ micelle density profiles where a reasonable 8 agreement was found between both water models. The aggregation number was one micelle in both with diameters of 3.2 and $3.0 \mathrm{~nm}$ for regular and polarizable water, respectively.

10 Simulations were carried out with the four Pluronics, 10R5, 31R1, L-35 and L-31 at several 11 concentrations, with and without the addition of SAILs. For the latter, we considered two 12 imidazolium compounds with different cation alkyl chain lengths, as well as one representative of 13 phosphonium SAILs. All possible combinations were tested with $1 \mathrm{wt} \%$ of Pluronic and $0.3 \mathrm{wt} \%$ 14 of $\left[\mathrm{C}_{n} \operatorname{mim}\right] \mathrm{Cl}(\mathrm{n}=10,14)$ and $\left[\mathrm{P}_{4,4,4,14}\right] \mathrm{Cl}$, at the cloud point temperatures determined 15 experimentally. In total, 16 MD simulations for different Pluronic/SAIL mixtures of at least 3.0 $\mu$ s of simulation time were carried out, the details of which can be consulted in Table S2. To more 17 clearly understand the complex interactions taking place during the initial stages of the self18 assembly of Pluronic/SAIL micelles, we have also carried out a series of MD simulations at room temperature $\left(25^{\circ} \mathrm{C}\right)$ for selected concentrations, as summarised in Table S3. For each Pluronic, these included the reference system without SAIL and two systems with $0.3 \mathrm{wt} \%$ of $\left[\mathrm{C}_{14} \mathrm{mim}\right] \mathrm{Cl}$ 21 or $\left[\mathrm{P}_{4,4,4,14}\right] \mathrm{Cl}$. The simulation times were also up to $3.0 \mu \mathrm{s}$.

22 All MD simulations were carried out with the Gromacs 5.1.4 package ${ }^{41}$ adopting the leapfrog 23 algorithm ${ }^{42}$ to integrate the equations of motion with a time step of $10 \mathrm{fs}$. The potential energy 24 function encompassed the bond stretching, angle bending and dihedral torsion for bonded 25 interactions, and Lennard-Jones (LJ) and Coulombic terms for non-bonded interactions. The 26 force-switch modifier was employed in the LJ interactions with the energy decaying smoothly to 27 zero between 1.0 and $1.2 \mathrm{~nm}$. The Verlet algorithm ${ }^{43}$ was used in combination with the neighbour 28 list updated every 20 steps. The cut-off function with the Potential-Shift-Verlet modifier was used 29 for long-range Coulombic interactions with a cut-off radius of $1.2 \mathrm{~nm}$. Long-range Coulombic 30 interactions were evaluated through particle mesh Ewald (PME). ${ }^{44}$ The 1.2 cut-off was selected 31 since the optimal PME mesh load for parallel simulations was below 0.5 and for highly parallel 
1 simulations this load should be between 0.25 and 0.33 , this circumstance can be amended by 2 slightly increasing the cut-off. All bonds were constrained by the LINCS algorithm. ${ }^{45}$ Cubic boxes 3 with periodic boundary conditions were used placing all the molecules randomly, followed by an 4 equilibrium process described previously. ${ }^{7}$ Afterwards, all systems were run for $0.5 \mu$ s of 5 simulation time in the $\mathrm{NpT}$ ensemble. Unless otherwise stated, the reported simulation time was

6 the number of steps multiplied by the nominal time step (10 fs). The temperature was fixed with

7 the velocity-rescaling thermostat ${ }^{46}$ in the equilibration stage and the Nose-Hoover thermostat ${ }^{47,48}$ 8 in the production runs, to ensure adequate ensemble sampling. The pressure coupling was 9 considered as isotropic and the pressure was fixed at 1 bar using the Parrinello-Rahman barostat. ${ }^{49}$ 10 The MD simulations were visualised using the VMD software package..$^{50}$ The density profile, 11 micelle distribution and the aggregation number were obtained using an in-house code ${ }^{7,51,52}$ based 12 on the Hoshen-Kopelman cluster-counting algorithm. ${ }^{53}$ 


\section{Normal}
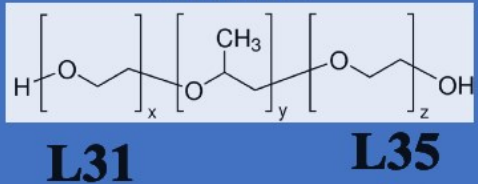

L35

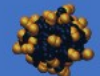

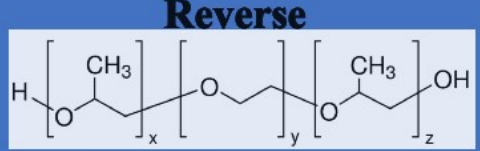

$31 \mathbf{R}$

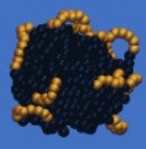

$10 \mathrm{R} 5$

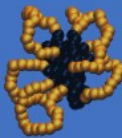

\section{SAILs}

a) 1-decyl-3-methylimidazolium chloride $-\left[\mathrm{C}_{10} \mathrm{mim}\right] \mathrm{Cl}$

b) 1-dodecyl-3-methylimidazolium chloride $-\left[\mathrm{C}_{12} \mathrm{mim}\right] \mathrm{Cl}$

c) 1-methyl-3-tetradecylimidazolium chloride - $\left[\mathrm{C}_{14} \mathrm{mim}\right] \mathrm{Cl}$

a)

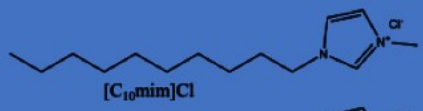

b)

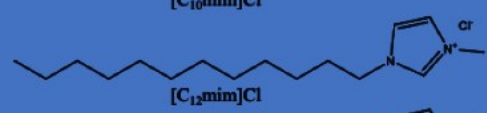

c)

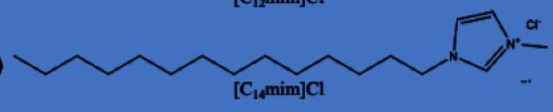

a) $\left[\mathrm{C}_{10} \min \right] \mathrm{Cl}$

b) $\mathrm{C}_{12} \operatorname{minCl}$

c) $\mathrm{C}_{14} \operatorname{minCl}$

Figure 1. Structure of the Pluronics and SAILs CG model used in this work. The Pluronic micelle pictures were taken from the MD simulations carried out previously. ${ }^{7}$ The PPG and PEG in the Pluronic micelles are in black and orange tributyltetradecylphosphonium chloride
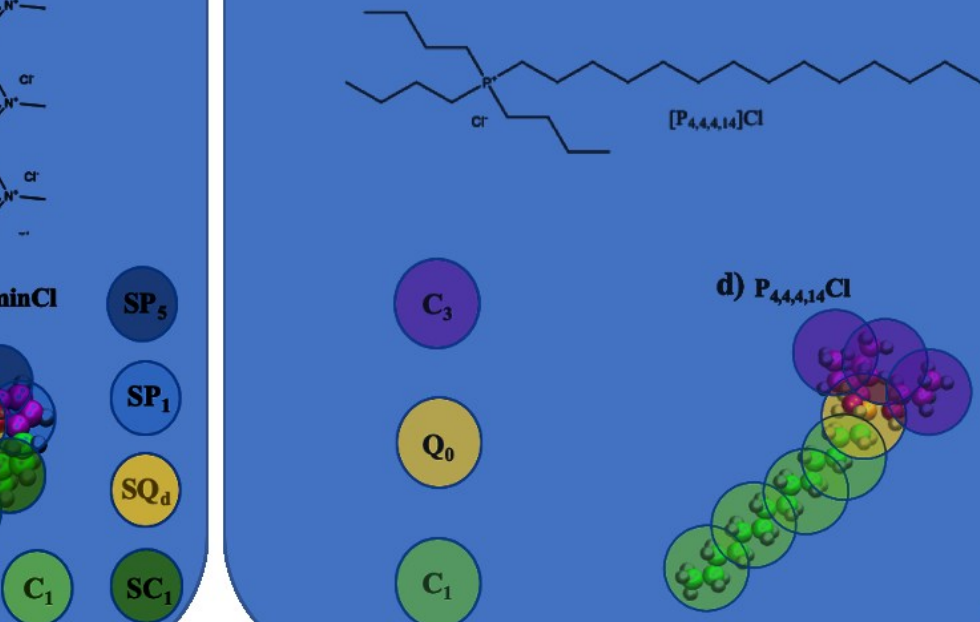
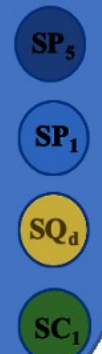
colour, respectively. In the bottom, the imidazolium-based ionic liquids were modelled by using a 3:1 mapping (3 heavy atoms per CG bead), labelled with the prefix $S$, with a size of $\sigma=0.43 \mathrm{~nm}$, whereas regular sized $\sigma=0.47 \mathrm{~nm}$ beads were used to map the phosphonium-based ionic liquid. The colour code is as follows; green is for the most apolar character, light orange for charged centers, purple for medium apolar behaviour, light blue for the lowest polar character and finally the dark blue colour was chosen for the most polar regions of the ionic liquid. 


\section{Results and discussion}

$\underline{3.1 \text { MD simulations of self-assembly in mixed Pluronic-SAIL systems }}$

In a previous paper, a new CG model was developed to provide a microscopic insight into the complex self-assembly of Pluronic surfactants in aqueous solutions. ${ }^{7}$ This CG model was our base line to study the impact of SAILs on the cloud point behaviour of Pluronic systems. Firstly, two Pluronics with the same amphiphilic character ( $50 \mathrm{wt} \%$ of PEG) were compared, namely the star-like L-35 and the flower-shape 10R5, to evaluate the effect of the micelle PEG surface shape.

8 Then, two normal Pluronics with the same PPG content but different PEG content, L-31 and L-35, were analysed to elucidate how the PEG moiety size affects the formation of mixed micelles. Finally, the reverse Pluronic 31R1 with $~ 10 \mathrm{wt} \%$ of PEG was also analysed and compared with 11 the 10R5, which has five times more EG units.

12 The room temperature simulations (see Table S3) provide an interesting picture of the initial stages 13 of self-assembly in these Pluronic/SAIL systems. To ensure that the micelle equilibrium is reached, 14 the total potential energy $\left(E_{t o t}\right)$ was monitored. The energy decreased during the initial micelle 15 formation until reaching a plateau as soon as the micellar growth stops taking place. After this, 16 only small fluctuations were observed as shown in Figure S3 for the Pluronic aqueous solutions 17 and their Surface-Active Ionic Liquid (SAIL) mixtures at 298K. This procedure was followed in 18 our previous study on Pluronic-water solutions, ${ }^{54}$ where the $\mathrm{E}_{\text {tot }}$ plateau region was reached after $193 \mu$ s of simulation time and the simulated micelle size distributions agreed with the results of 20 experimental measurements. This suggests that the system had been successfully equilibrated and 21 gives us confidence that the same is true for the simulations incorporating SAIL in the Pluronic 22 aqueous solutions. Figure $\mathbf{S 3}$ emphasizes that the equilibrium was attained after $1 \mu$ s in all of the 23 systems except 31R1 which required $2 \mu$ s due to the high molecular weight (this system includes $24 \sim 1.5$ million CG water beads). Additionally, to ensure that our simulations are free from any 25 system-size effects, the 10R5 systems containing 60 Pluronic moieties (twice the normal size) were 26 simulated over $3 \mu$ s for the Pluronic aqueous solutions and their SAIL mixtures, concretely with $27\left[\mathrm{P}_{4,4,4,14}\right] \mathrm{Cl}$ and $\left[\mathrm{C}_{14} \mathrm{mim}\right] \mathrm{Cl}$. Table $\mathbf{S 4}$ shows the number of micelles, the aggregation number and 28 micelle diameters for those simulations. Very good agreement was found between small and large 29 systems. Additionally, the double-sized 10R5/water system was run for $9 \mu$ s, and we observed that 30 the micelle distribution was unchanged between $4 \mu$ s and $9 \mu$ s. The $E_{\text {tot }}$ for the double-sized 31 systems was monitored and compared with results obtained with the small systems. Both systems 
1 followed a similar pattern, with the smaller system reaching equilibrium after $\sim 1 \mu$ s (solid lines)

2 whereas the double-sized systems (dashed lines), required $2 \mu$ s at least, as shown in Figure S4. To

3 further ensure that the micelle distribution equilibrium was reached the aggregation number $\left(\mathrm{N}_{\mathrm{a}}\right)$

4 vs simulation time is shown in Figure $\mathbf{S} 5$ for all systems at $25^{\circ} \mathrm{C}$. It can be seen that between 2 and

$53 \mu \mathrm{s}$, the $\mathrm{N}_{\mathrm{a}}$ barely changes, reaching a Plateau, which suggest that micelles have reached their

6 equilibrium size distribution.

7 The final simulation snapshots are shown in Figure 2, displaying the micelle formation with 8 and without the addition of SAIL. It can be clearly seen that in the presence of SAIL, hybrid 9 micelles containing both Pluronic and SAIL molecules were formed. This is because both 10 components have amphiphilic properties and at these dilute conditions, both have a tendency to 11 form micellar aggregates. A key difference between the two amphiphiles is that SAIL molecules 12 are charged, while Pluronics are neutral. As we will discuss shortly, this introduces a Coulombic 13 character into the otherwise neutral Pluronic micelles, with some interesting consequences on the 14 cloud point behaviour. 

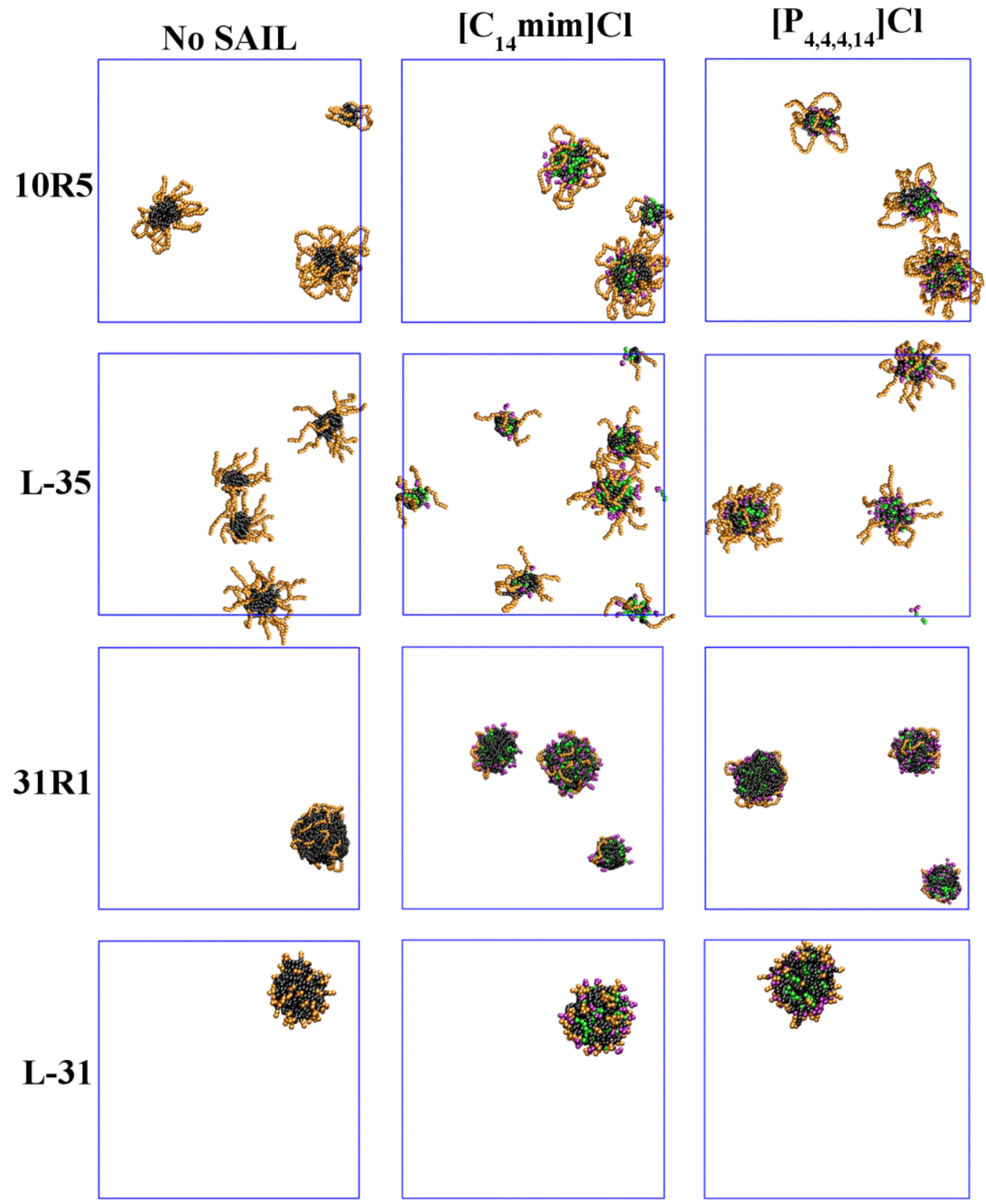

Figure 2. MD simulation snapshots after $3.0 \mu$ s of simulation time for $10 \mathrm{R} 5, \mathrm{~L}-35,31 \mathrm{R} 1$ and $\mathrm{L}-31$ at $1 \mathrm{wt} \%$ in aqueous 3 solutions (left column) and their mixtures with $0.3 \mathrm{wt} \%$ of $\left[\mathrm{C}_{14} \mathrm{mim}\right] \mathrm{Cl}$ and $\left[\mathrm{P}_{4,4,4,14}\right] \mathrm{Cl}$, (middle and right columns, 4 respectively). The temperature was fixed to $25^{\circ} \mathrm{C}$. The colour code is as follows: for Pluronics, the PPG and PEG are 5 in black and orange, respectively; for SAILs, the hydrophobic alkyl-chain tail is green whereas the imidazolium ring in $\left[\mathrm{C}_{\mathrm{n}} \mathrm{mim}\right] \mathrm{Cl}$ and the butyl head groups in $\left[\mathrm{P}_{4,4,4,14}\right] \mathrm{Cl}$ are coloured in purple. Water and chloride ions were removed 7 for clarity. 
Given the amphiphilic properties of both components, it is interesting to examine the mechanism of micelle self-assembly in more detail. The self-assembly processes can be followed closely in the MD simulation movies SM1 to SM4 (one for each Pluronic) which can be found in the Supporting Information. In Figure 3, we show several snapshots of the initial stages of equilibration in solutions of 10R5 Pluronic, with and without SAIL. We focused on the two classes of ionic liquids, imidazolium and phosphonium, with the same alkyl chain length. As previously described, ${ }^{7}$ in the solution without SAIL, individual 10R5 molecules start by forming small loops whereby the two hydrophobic PPG ends join together. Shortly after that, the loops aggregate into small micelles, which eventually fuse together to form the equilibrium micelle distribution. When SAILs are present in the solution (middle and right columns in Figure 3), the 10R5 loops coexist with small aggregates (proto micelles) of the SAIL that are also formed quickly at the beginning of the simulation. As the self-assembly progresses, some of the isolated Pluronic molecules are incorporated in growing SAIL aggregates, and vice-versa. This originates two classes of hybrid micelles, one dominated by Pluronics with some adsorbed SAIL (we call these Pluronic/SAIL micelles) and another class dominated by SAIL with some adsorbed Pluronics (i.e. SAIL/Pluronic micelles). Subsequent micelle fusion gives rise to a small number of larger hybrid micelles that contain both amphiphilic components, as shown for 10R5 $+\left[\mathrm{C}_{14} \mathrm{mim}\right] \mathrm{Cl}$ and $10 \mathrm{R} 5+\left[\mathrm{P}_{4,4,4,14}\right] \mathrm{Cl}$ in Figure 2.

The self-assembly mechanism described above is similar, regardless of the type of SAIL that is present. This can be seen by comparing the middle and right columns in Figure 3. The main difference is that the initial SAIL aggregates are somewhat larger for $\left[\mathrm{P}_{4,4,4,14}\right] \mathrm{Cl}$ than for $\left[\mathrm{C}_{14} \mathrm{mim}\right] \mathrm{Cl}$, due to the slightly more hydrophobic head of the former. Furthermore, the mechanism is very similar for L-35 (Figure S6), except for the fact that the Pluronic is normal and not reverse, 24 thus the chains initially form "knots" instead of loops - the effect of SAIL is, however, qualitatively the same. Figure $\mathbf{S} 7$ shows a close-up for this system, where we can easily discern individual SAIL and Pluronic micelles formed in the early stages of self-assembly. Hybrid SAIL/Pluronic and Pluronic/SAIL aggregates can also be discerned. It is important to note that for both 10R5 and L-35 systems, the evolution of the average aggregation number with time is very similar in the solutions with and without SAIL (Figures 3, S5 and S6) - at any given time, there is approximately the same number of micelles in the three solutions. 


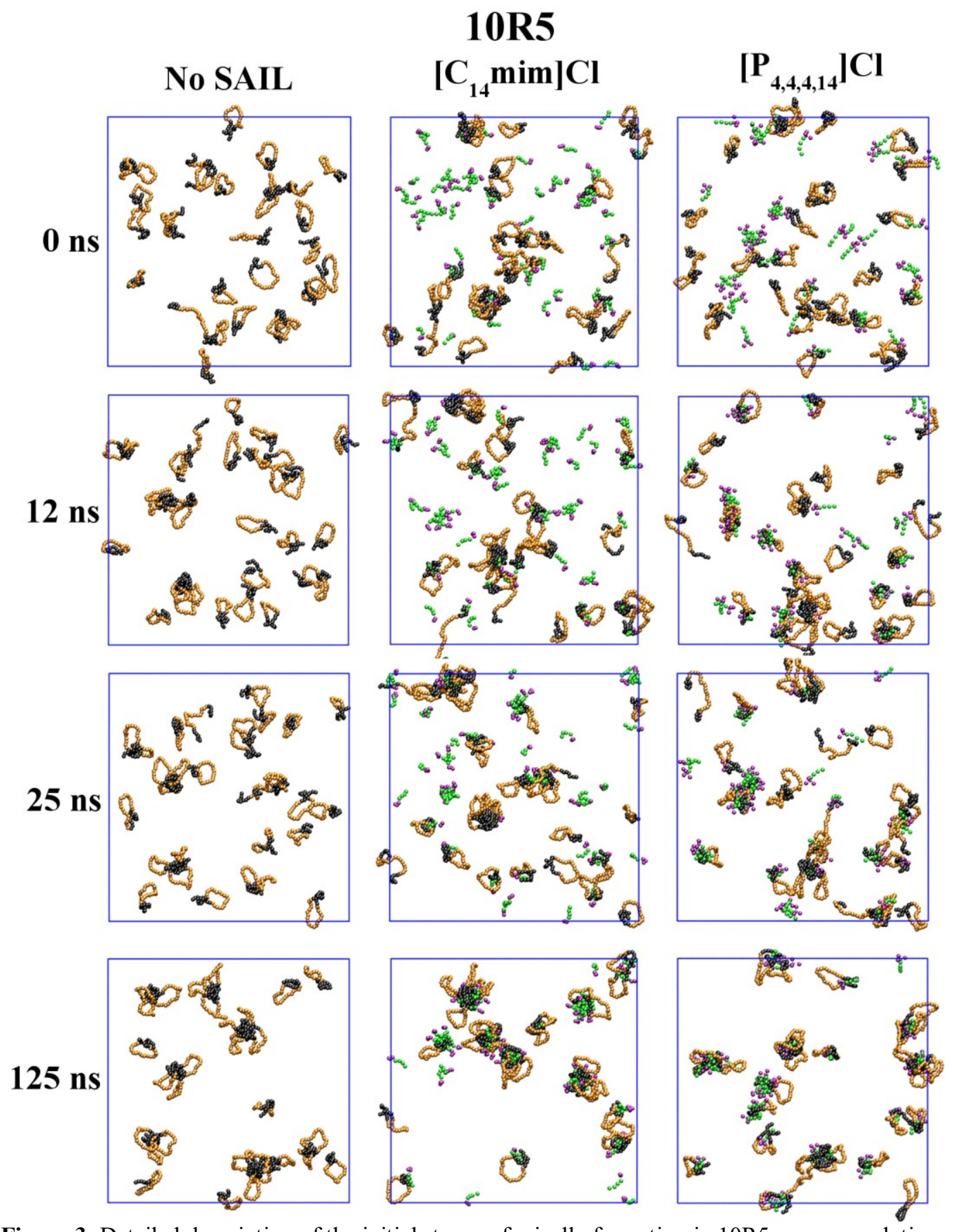

Figure 3. Detailed description of the initial stages of micelle formation in 10R5 aqueous solutions (left column) and their mixtures with $\left[\mathrm{C}_{14} \mathrm{mim}\right] \mathrm{Cl}$ (middle column) and $\left[\mathrm{P}_{4,4,4,14}\right] \mathrm{Cl}$ (right column). The temperature was fixed to $25^{\circ} \mathrm{C}$. The colour code is the same as in Figure 2. 
This picture changes significantly when we consider the two Pluronics with larger PPG 2 segments, 31R1 (Figure 4) and L-31 (Figure S8). In these cases, the Pluronic self-assembly in the 3 reference system without SAIL takes place much faster, due to the large driving force for 4 aggregation caused by the long PPG chains. As a consequence, large Pluronic micelles are already 5 formed after a few tens of nanoseconds (see left column of Figure 4). In contrast, this does not

8 followed by much slower processes of micelle growth and micelle fusion. In fact, even after 125

9 ns, there are still micelles of pure SAIL in the solution (see bottom-right panel of Figure 4). One

10 can conclude that one of the effects of the addition of SAIL in these systems is that micelle fusion

11 becomes much less favourable, which slows down the self-assembly process.

12 In Figure 5, we show a more detailed picture of the hybrid micelles formed in the 13 Pluronic/SAIL aqueous solutions. As we can see, the Pluronic/SAIL micelles have quite similar 14 structures to the pure Pluronic micelles (compare with the examples shown in Figure 1 and those 15 previously reported). ${ }^{7}$ The ionic liquid molecules are embedded within the PPG core, with the 16 charged head groups protruding into the PEG corona region. The SAIL/Pluronic micelles (right17 hand side of Figure 5) also show a similar structure to pure SAIL micelles, ${ }^{33,55}$ with a hydrophobic 18 core composed of the alkyl chains and most of the polar heads at the surface. In this case, the PPG 19 moieties of the Pluronics are embedded in the alkyl core, while the hydrophilic PEG chains 20 protrude outward into the aqueous solution. It is also apparent that the arrangement of the Pluronic 21 chains in the SAIL/Pluronic micelles is rather disordered, particularly when compared to the pure 22 Pluronic micelles. 


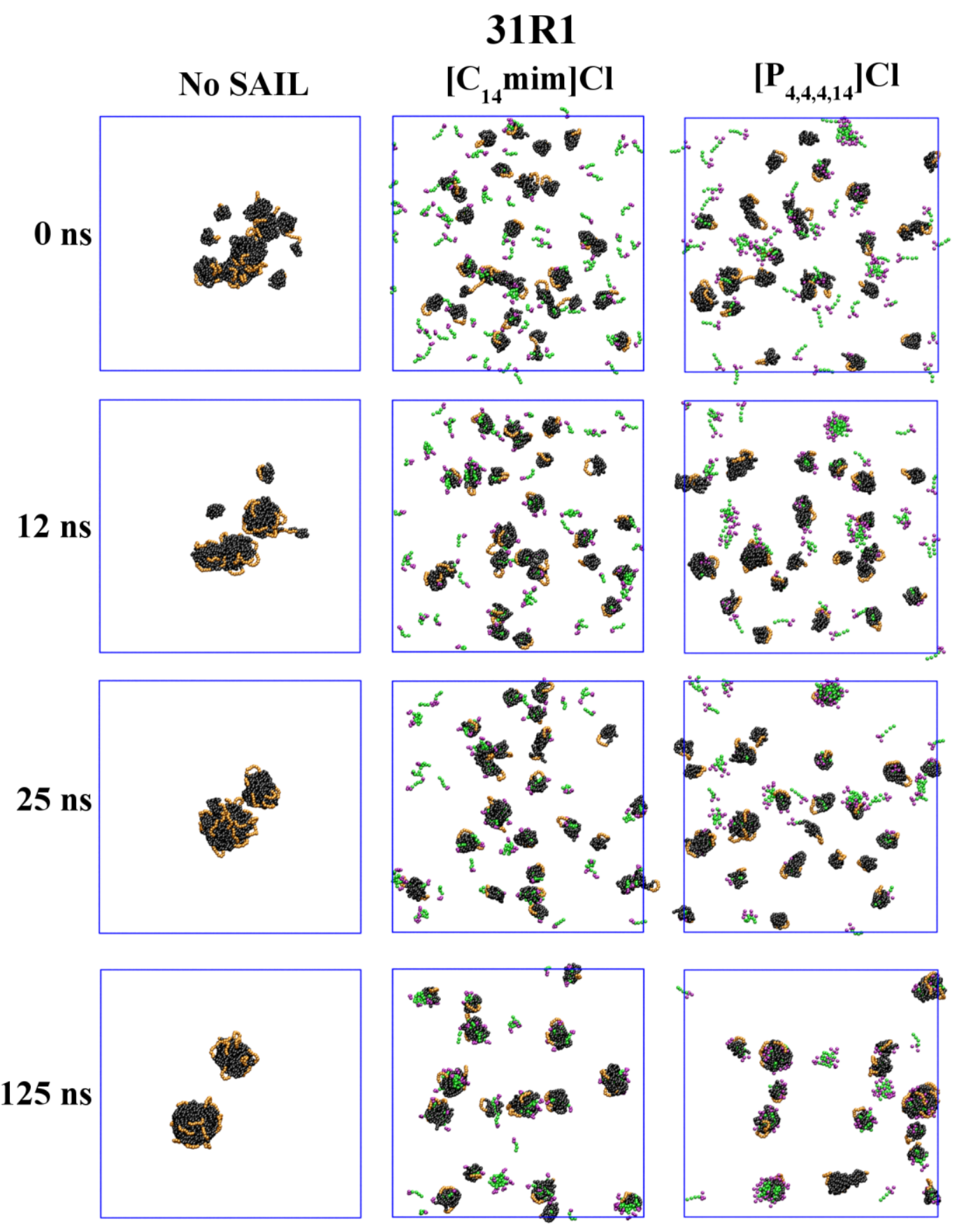

Figure 4. Detailed description of the initial stages of micelle formation in 31R1 aqueous solutions (left column) and their mixtures with $\left[\mathrm{C}_{14} \mathrm{mim}\right] \mathrm{Cl}$ (middle column) and $\left[\mathrm{P}_{4,4,4,14}\right] \mathrm{Cl}$ (right column). The temperature was fixed to $25^{\circ} \mathrm{C}$. The colour code is the same as Figure 2. 


\section{Pluronic/SAIL SAIL/Pluronic}

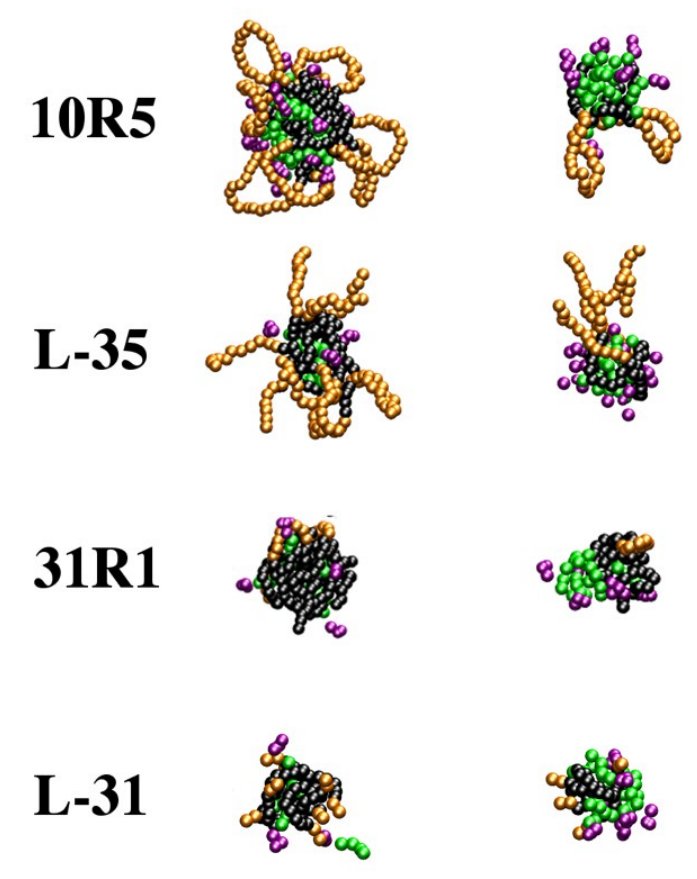

Figure 5. Examples of hybrid Pluronic/SAIL and SAIL/Pluronic micelles found in the MD simulations for 10R5, L-35, 31 R1 and L-31 Pluronics mixed with SAILs. The colour code is the same as in Figure 2.

5 As mentioned before, the incorporation of SAIL molecules into the Pluronic micelles introduces 6 a charged character at the micelle surface. This can be seen in the average radial density profiles 7 of the Pluronic/SAIL micelles, an example of which, for the L-31 Pluronic, is shown in Figure 6. 8 Similar density profile plots for the other 3 systems can be found in the Supporting Information 9 (Figures S9-S11). In all cases, the charged regions of the SAIL cations arrange themselves near 10 the interface between the PPG core and the PEG corona. The chloride counterions, on the other 11 hand, arrange themselves in a diffuse layer that is mostly outside the core of the micelle, as 12 observed for typical cationic surfactant micelles. ${ }^{51}$ This means that inter-micellar repulsion due to 13 the electrical double layer will hinder micelle fusion, which explains why this process was so much 14 slower in Pluronic/SAIL solutions (Figure 4). This charged character of the mixed micelles will 15 also have a pronounced effect on the onset of the cloud point, as discussed in section 3.2. 

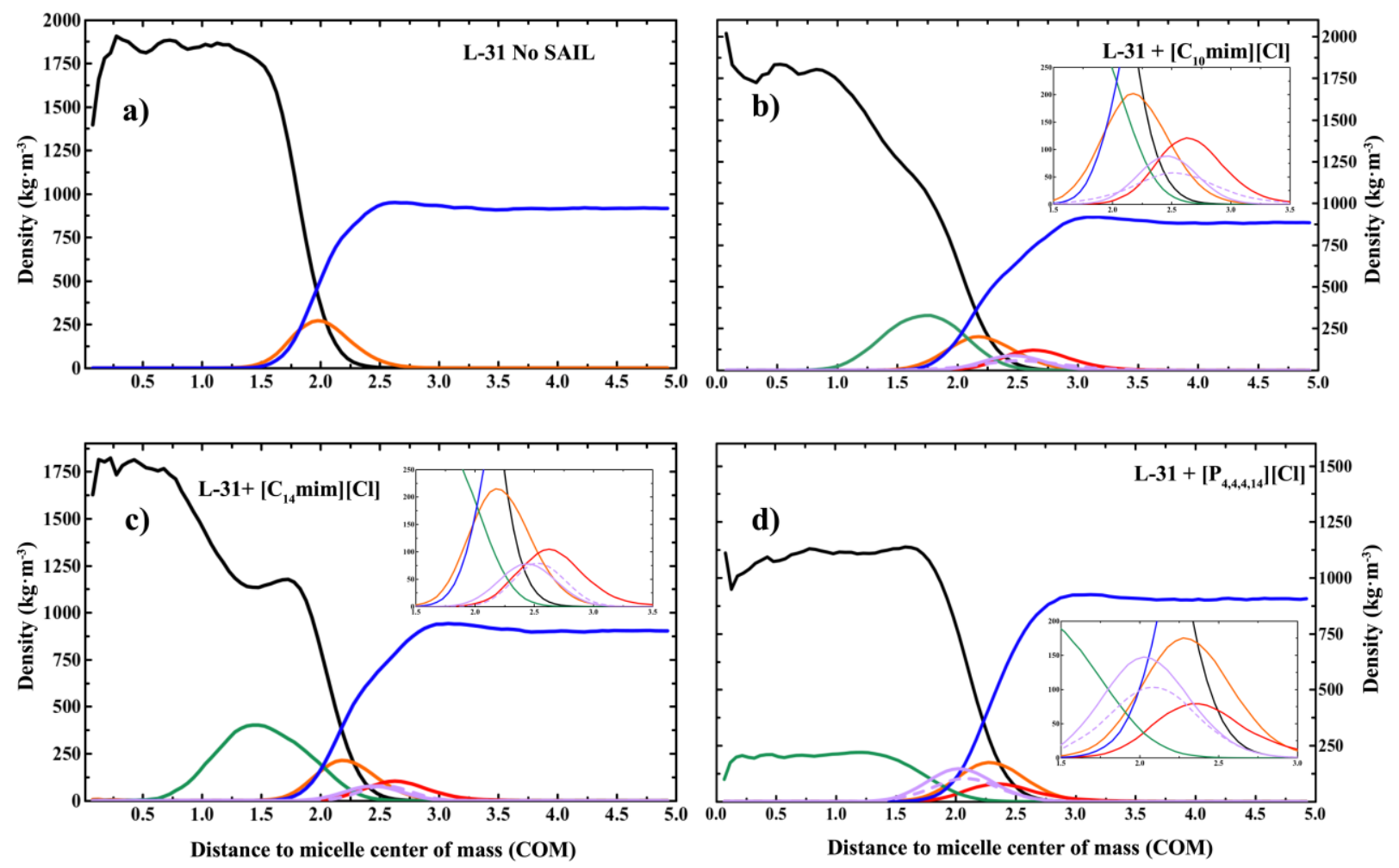

Figure 6. Micelle density profiles for L-31 solutions without SAILs (a) and after addition of $\left[\mathrm{C}_{10} \mathrm{mim}\right] \mathrm{Cl}(\mathrm{b})$, $3\left[\mathrm{C}_{144 \mathrm{mim}}\right] \mathrm{Cl}(\mathrm{c})$ and $\left[\mathrm{P}_{4,4,4,14}\right] \mathrm{Cl}(\mathrm{d})$. The colour code is as follows: SAIL alkyl-chain tails in green, SAIL head groups 4 in purple, water in blue, chloride counter ions in red, PEG micelle corona in orange and PPG micelle hydrophobic core in black. The dashed purple line shows the profile for the charged site of the SAIL head group, multiplied by a factor of 3 for ease of visualization. The insets show a blow up of the head-group region for the systems with SAIL.

Interestingly, there are some subtle structural differences in micelles formed with different SAILs. The much bulkier nature of the phosphonium head group gives it a somewhat more 10 hydrophobic character. As a consequence, the corresponding peaks tend to be on the inner side of 11 the micelle surface, closer to the PPG core (compare the position of the purple lines in the insets 12 of Figure 6). In particular, the charged site peak is well to the left of the peak corresponding to the 13 PEG corona, indicating that it is somewhat "buried" within the core. In contrast, the smaller, and 14 more hydrophilic, imidazolium head groups tend to protrude further into the PEG corona, being 15 more exposed to the solution medium. In fact, for both imidazolium cations, the peak of the 16 charged group profile is to the right of the PEG corona, and well outside of the PPG core. This 17 observation is confirmed by examining Surface Distribution Functions (SDF) of the charged bead 18 of each SAIL around the interface between the PEG and PPG groups of the Pluronic (Figure 7). 
1 These SDFs essentially show the average surface of charge around each Pluronic molecule in a

2 hybrid micelle. As can be seen, the surface moves closer to the PPG groups with the phosphonium

3 SAIL when compared to the imidazolium counterpart with the same chain length (Figures 7a and

4 7b). This means that systems containing imidazolium will exhibit more pronounced effects of 5 inter-micellar repulsion. The same structural differences were observed for all other Pluronic 6 systems (see Figures S9-S11). However, the effect is more pronounced, in relative terms, for the 7 Pluronics with less PPG and more PEG because the micelles become significantly smaller and the 8 adsorption of SAIL leads to a larger perturbation of the surface structure.
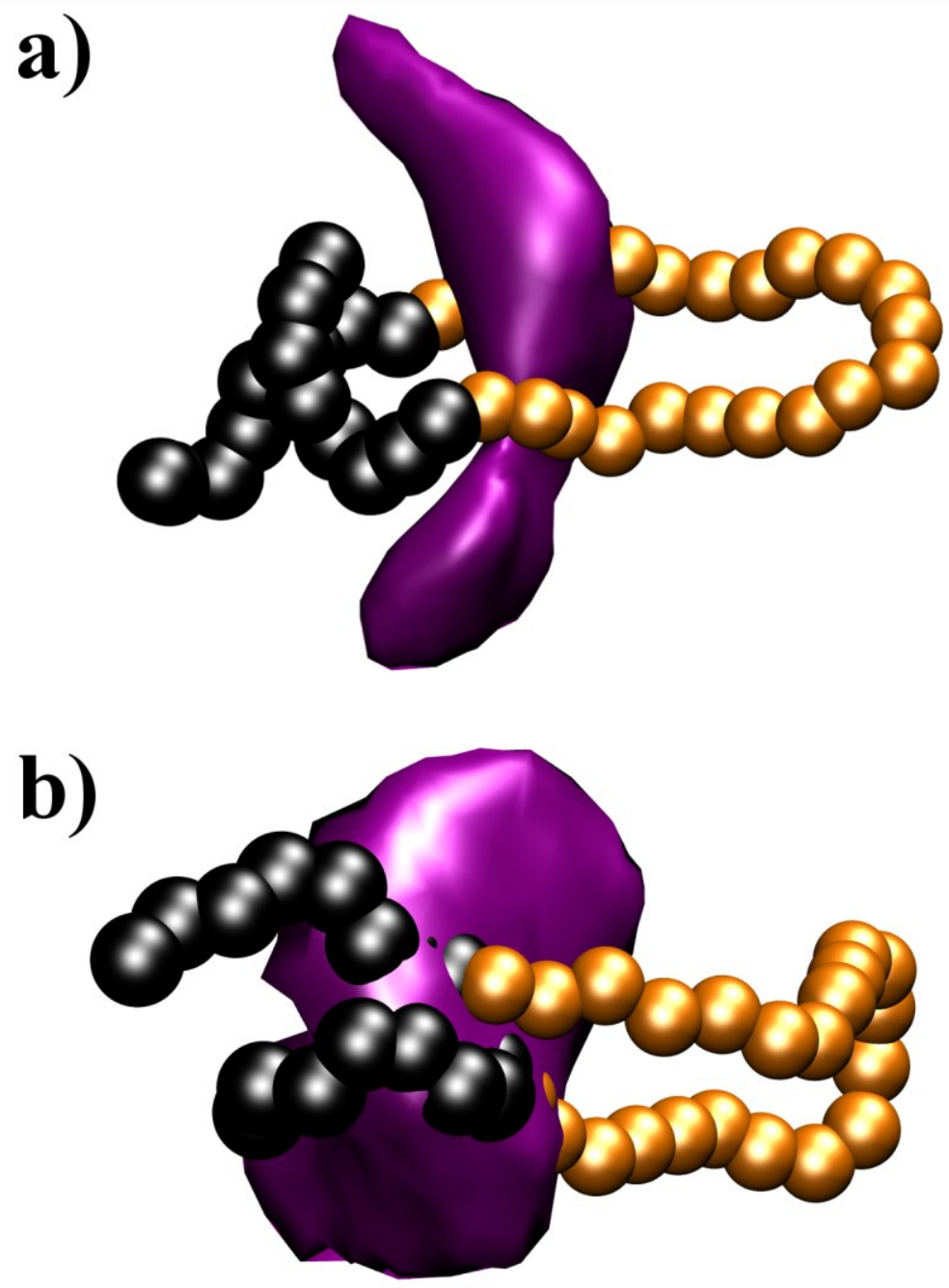

Figure 7. Surface Distribution Functions for the charged IL cation site with respect to the PEG to PPG interface 12 in the 10R5 system with: (a) $\left[\mathrm{C}_{14} \mathrm{mim}\right] \mathrm{Cl}$; (b) $\left[\mathrm{P}_{4,4,4,14}\right] \mathrm{Cl}$. The micelle interface was defined by considering the three 13 PEG beads closest to the PPG group as a reference for calculating the SDFs. An example configuration of a single 
Pluronic chain is also shown for comparison. The PEG sites are in orange, the PPG in black and the cation charged site probability surface in purple.

Comparing micelles containing $\left[\mathrm{C}_{10} \mathrm{mim}\right] \mathrm{Cl}$ and $\left[\mathrm{C}_{14} \mathrm{mim}\right] \mathrm{Cl}$, the average location of the head group is nearly the same. However, the $\left[\mathrm{C}_{10} \mathrm{mim}\right] \mathrm{Cl}$ system shows a much broader distribution, suggesting that the arrangement of the SAIL at the micelle surface is significantly more disordered than in the case of $\left[\mathrm{C}_{14} \mathrm{mim}\right] \mathrm{Cl}$. This suggests that the effects of inter-micellar repulsion will not be as pronounced with the shorter-chain IL cation. Interestingly, this difference was only significant for the L-31 Pluronic -for the other three systems, the $\left[\mathrm{C}_{10} \mathrm{mim}\right] \mathrm{Cl}$ and $\left[\mathrm{C}_{14} \mathrm{mim}\right] \mathrm{Cl}$ are almost indistinguishable (Figures S6-S8). This is possibly due to the extremely small PEG corona

11 in L-31, which makes it harder for the shorter IL cation to adopt a very ordered arrangement at the 12 surface. in aqueous solutions. ${ }^{7}$ The PPG:PEG ratio plays an important role in controlling the cloud point,

17 since it determines the balance between hydrophobic and hydrophilic interactions. For example, 18 when the PPG:PEG ratio is increased from 10R5 to 31R1, the micelles become much larger due to the hydrophobic interactions being stronger than the hydrophilic ones, and this leads to a much lower cloud point temperature. ${ }^{7}$ Furthermore, the differences in structure between normal (PEGPPG-PEG) and reverse (PPG-PEG-PPG) Pluronics induce major micelle surface changes from star-like to flower-like shaped micelles, respectively ( $c f$. Figure 1). In reverse Pluronics, above a certain concentration, the possibility of physical cross-linking between micelles favours selfaggregation, thus, the system requires less energy to coalesce and separate into two macroscopic phases than the corresponding normal Pluronic. This is the reason behind the lower cloud points observed in Pluronic 10R5 when compared with L-35, both with the same PEG and PPG content $27(50 / 50 \%)$.

28 The complex interactions between non-ionic Pluronics and SAIL cations were studied alongside the binodal curves. Above $3 \mathrm{wt} \%$ Pluronic concentration, for the low concentration of SAIL $(0.3 \mathrm{wt} \%)$ used in this work, the co-polymer totally dominates the phase behaviour resulting 
1 dilute systems since these display the greatest variation in the cloud points with SAIL 2 concentration and type. Figure 8 summarizes the experimental cloud points for $<5$ wt $\%$ of 3 Pluronic concentration with $0.3 \mathrm{wt} \%$ of SAIL. Imidazolium-based SAILs are represented by $4 \quad\left[\mathrm{C}_{\mathrm{n}} \operatorname{mim}\right] \mathrm{Cl}(\mathrm{n}=10,14)$ and phosphonium-based SAIL by $\left[\mathrm{P}_{4,4,4,14}\right] \mathrm{Cl}$. This selection was made 5 based on previous results in which these SAILs increased the cloud point temperature of non-ionic surfactant mixtures, albeit in markedly different ways. ${ }^{30,31}$
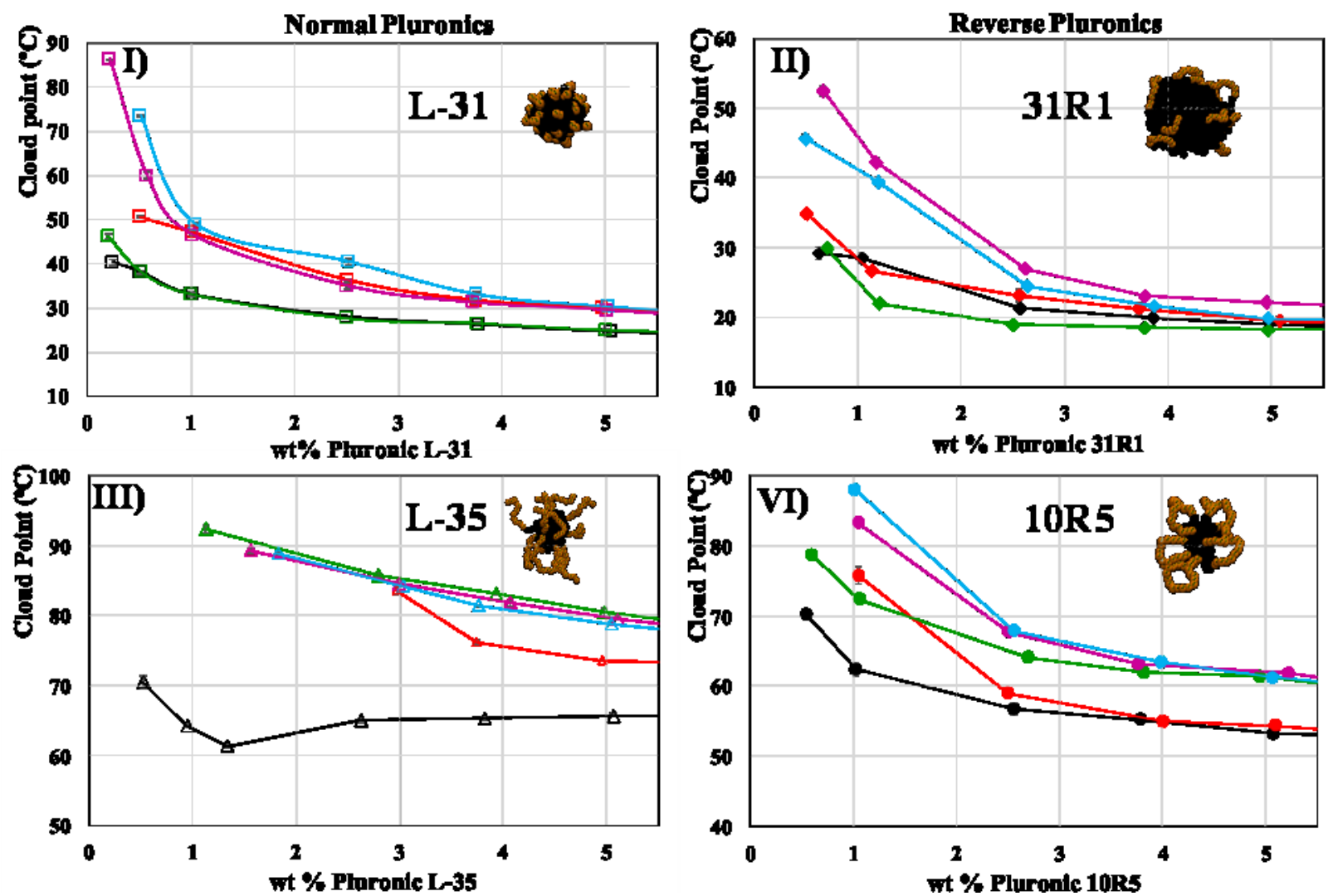

Figure 8. Binodal curves of Pluronic copolymers $+0.3 \mathrm{wt} \%$ of SAIL $+0.18 \mathrm{M}$ of McIlvaine buffer $\mathrm{pH}$ 7.0. I) $\square$, Pluronic L-31. II) $\Delta$, Pluronic L-35. III) ^, Pluronic 31R1. IV) •, Pluronic 10R5. The SAILs added to the Pluronic aqueous solutions are:,$-\left[\mathrm{C}_{10} \mathrm{mim}\right] \mathrm{Cl} ;-,\left[\mathrm{C}_{12} \mathrm{mim}\right] \mathrm{Cl} ;-,\left[\mathrm{C}_{14} \mathrm{mim}\right] \mathrm{Cl} ;-,\left[\mathrm{P}_{4,4,4,14}\right] \mathrm{Cl}$. The black line corresponds to the reference system without SAIL. The error bars are smaller than the symbol size. The inset snapshot micelles were taken from our MD simulations.

13

14

In general, the addition of SAIL induces an increase in the Pluronic cloud points, though this effect seems to be dependent on the Pluronic structure. The changes in these cloud points differ from those observed in previous works for mixed AMTPS composed of conventional non-ionic surfactants, namely belonging to the polyoxyethylene alkyl ether family, and SAILs of the $\left[\mathrm{C}_{\mathrm{n}} \mathrm{mim}\right] \mathrm{Cl}$ family with $\mathrm{n}<8$, for which the cloud point increased with the decrease of the SAIL 
1 alkyl side chain length. ${ }^{30,31,56}$ In this work, $\left[\mathrm{C}_{10}\right.$ mim $] \mathrm{Cl}$ displayed the lowest impact on the cloud 2 points, in some cases with no influence at all (see, e.g. the Pluronic L-31 binodal curve).

3 Conversely, $\left[\mathrm{C}_{10} \mathrm{mim}\right] \mathrm{Cl}$ and $\left[\mathrm{C}_{14} \mathrm{mim}\right] \mathrm{Cl}$ both behaved similarly, exhibiting the highest cloud

4 point temperatures. The $\left[\mathrm{P}_{4,4,4,14}\right] \mathrm{Cl}$ also induces an increase on the cloud points albeit lower than

5 imidazolium with similar alkyl-chain length $\left(\left[\mathrm{C}_{14} \mathrm{mim}\right] \mathrm{Cl}\right) .{ }^{31}$

6 The experimental cloud point conditions shown in Figure 8 were reproduced by MD 7 simulations to analyse the thermo-response behaviour of these Pluronic/SAIL mixtures. The 8 Pluronic concentration was $1 \mathrm{wt} \%$ since the experimental results exhibited the greater effect with 9 a SAIL concentration of $0.3 \mathrm{wt} \%$. The MD simulation snapshots of the overall systems (see Table $10 \mathbf{S 2}$ for details) after $3.0 \mu$ s are shown in Figure 9. A detailed view of the micelle formation is 11 shown in the simulation movies (Films SM5-SM14; see Supporting Information) of the 12 Pluronic/SAIL systems corresponding to the snapshots of Figure 9. 

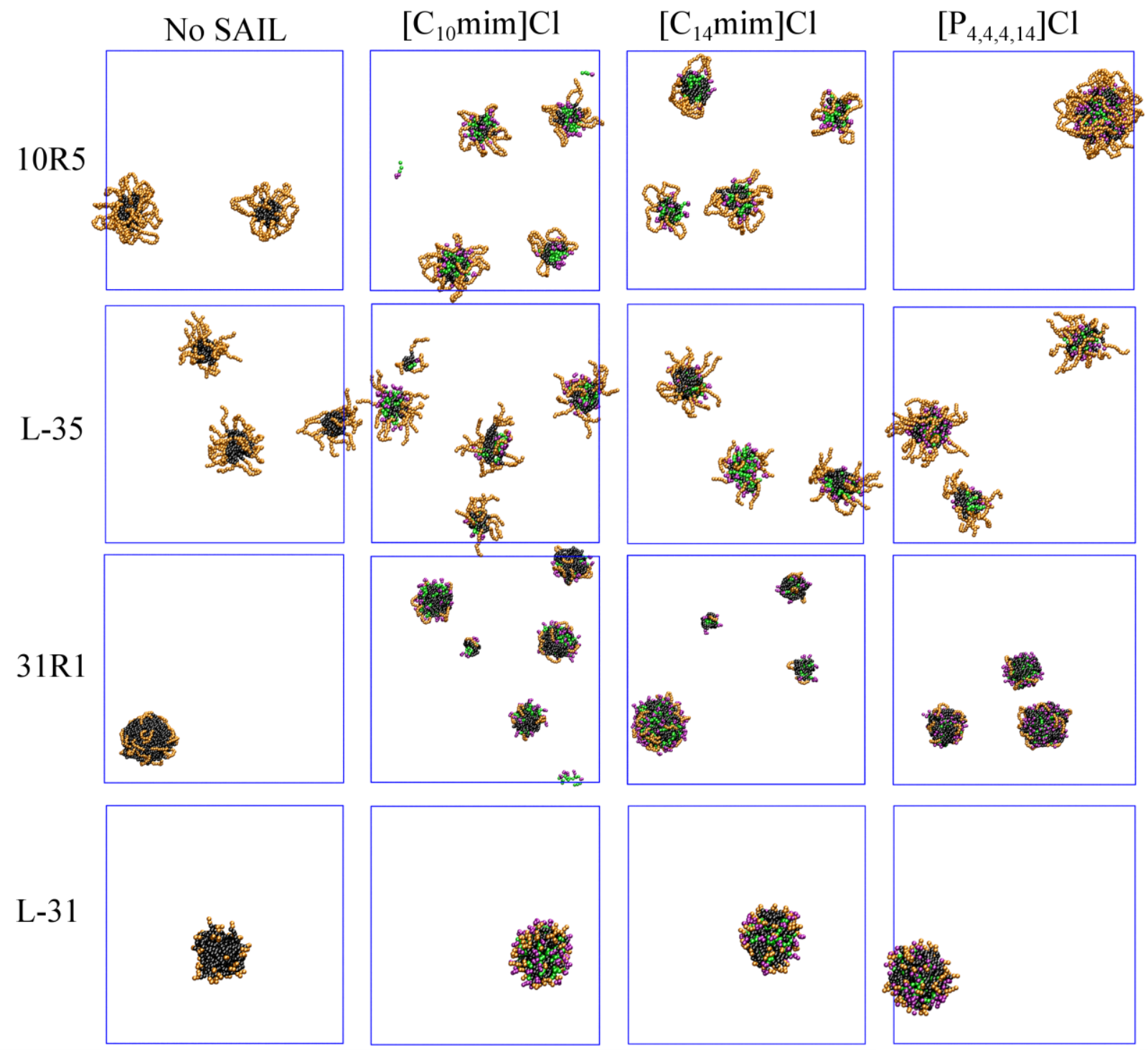

Figure 9. MD simulation snapshots after $3.0 \mu$ s for Pluronics 10R5, L-35, L-31 and 31R1 at $1 \mathrm{wt} \%$ in aqueous solutions (top row) and their mixtures with $0.3 \mathrm{wt} \%$ of $\left[\mathrm{C}_{10} \mathrm{mim}\right] \mathrm{Cl},\left[\mathrm{C}_{14} \mathrm{mim}\right] \mathrm{Cl}$ and $\left[\mathrm{P}_{4,4,4,14}\right] \mathrm{Cl}$ (3 lower rows). The temperature was set at the experimental cloud point temperatures shown in Figure 8 and also displayed in Table S2.

5 The colour code is the same as in Figure 2.

The competition between Pluronic and SAIL micelle formation as well as the adsorption of the 8 second compound into the formed micelles leads to a rather complex behaviour. It is the balance 9 between each of the individual effects identified in section 3.1 that leads to the different cloud 10 point curves for each system. First of all, with few exceptions, addition of SAIL to an aqueous 11 solution of Pluronics leads to an increase in the cloud point. This is generally caused by the 
1 introduction of surface charge (see, e.g., Figure 7), which leads to inter-micellar repulsions and

2 makes it more difficult for micelles to aggregate. As a consequence, the onset of the cloud point is 3 raised up to higher temperatures.

4 Another general observation is that phosphonium cations have less impact on the cloud point 5 than imidazolium cations of the same chain length. In fact, the red lines (for phosphonium) in

6 Figure 8 tend to be located at lower temperatures than the blue lines, corresponding to the 7 imidazolium cation with the same chain length. This shift in temperature with the type of head 8 group is more pronounced for the smaller Pluronic solutions (lower panels in Figure 8). This shift is mainly due to the different effects on the micelle structure induced by these two cations. As explained earlier, the phosphonium charged site is much more "buried" inside the PPG core, 11 whereas the smaller imidazolium head group protrudes much more onto the PEG corona. As a consequence, Pluronic micelles with adsorbed imidazolium are more prone to inter-micellar 13 repulsion, resulting in a stronger effect on the cloud point. Furthermore, as seen in Figure 9, the 14 systems with $\left[\mathrm{P}_{4,4,4,14}\right] \mathrm{Cl}$ tend to form larger micelles at the CPT compared with $\left[\mathrm{C}_{14} \mathrm{mim}\right] \mathrm{Cl}$, which 15 leads to a more diffuse surface charge.

16 The surface effects are less pronounced in Pluronics with larger PPG cores like L-31 and 31R1 17 than in the Pluronics with smaller PPG cores, such as L-35 and 10R5. This is because the onset of 18 the cloud point in the former is much more dominated by hydrophobic effects, so surface effects 19 are less important in relative terms. In the particular case of the L-31 system, both types of SAIL 20 seem to have similar effects on the cloud point (see Figure 8I). In this case, as seen in Figure 9, 21 the presence of SAIL has no marked effect on the micelle size, with all solutions equilibrating to 22 a single large micellar aggregate. Furthermore, the PEG corona in L-31 is extremely small, which 23 means that even the phosphonium charged groups are rather exposed to the solution. Taken 24 together, this means that the L-31 system is the least sensitive to structural differences caused by 25 changing the head group type.

26 Changing the alkyl chain length of the imidazolium cations also has different effects depending 27 on the type of Pluronic system. For the smaller Pluronics, all the imidazolium cations induce nearly 28 the same increase in the cloud point, regardless of the chain length (at least for chains larger than 2910 carbons). In contrast, for the larger Pluronics, $\left[\mathrm{C}_{10 \mathrm{mim}}\right] \mathrm{Cl}$ has very little effect on the cloud 30 point temperature. Once again, this can be partly explained by the balance between hydrophobic 31 and electrostatic interactions in the mixed systems. For the small Pluronics, even the small 
$1 \quad\left[\mathrm{C}_{10 \mathrm{mim}}\right] \mathrm{Cl}$ cation is able to accumulate at the surface and induce pronounced inter-micellar 2 repulsion. In the larger Pluronics, in particular for L-31, the smaller cation shows a much less 3 ordered arrangement at the surface, leading to weaker electrostatic repulsion. In addition, the 4 hydrophobic driving force for aggregation is much stronger, and effectively dominates the onset 5 of the cloud point.

We observed that at higher temperatures, the micelle PEG coronas are somewhat dehydrated, which facilitates micelle fusion processes; However, this was not always the case Actually, 8 depending on the concentration, some Pluronic aqueous solutions can exhibit a weak temperature impact in the micelle distribution ${ }^{57}$ as also noticed by comparing the results shown in Figures 2 and 9. Table S5 displays the characteristics of the micelle distributions obtained in the MD 11 simulations at CPT for all systems. The L-31 and 31R1, both with relatively high PPG content, 12 showed a nearly temperature-independent micellar distribution. However, the 10R5 and L-35, with 13 similar PEG:PPG ratios, exhibited $3 / 4$ micelles at $25^{\circ} \mathrm{C}$, and $2 / 3$ micelles at the CPT. Furthermore, 14 the addition of SAILs produced a different impact in the micelle distribution which also depends 15 on their nature, as discussed in more detail below. Additionally, all systems considered in this 16 work were run without Pluronics, i.e., as SAIL aqueous solutions, and no more than one or two 17 SAIL micelles were found in all of the cases. Thus, it is clear that the interaction between Pluronic 18 and SAIL plays an important role affecting the micelle distribution in the mixed systems when compared with their respective aqueous solutions.

20 It becomes clear from the micellar growth profiles shown in Figure $\mathbf{S 5}$ and the final micellar 21 distribution displayed in Table S5 that Pluronics become more thermo-responsive in the presence 22 of SAILs, except in the L-31 and 31R1 cases, where the relatively high PPG content dominates 23 the aggregation as it can be also noticed in the simulation snapshots displayed in Figures 2 and 9.

24 This fact was also confirmed in the CPT curves of L-31 and 31R1 obtained in the experiments 25 (Figure 8). The impact of SAILs observed in 10R5 and L-35, led us to conclude that the less 26 screened charge and more hydrophilic head-group of imidazolium in Pluronic micelles somehow 27 hinders the micelle growth.

28 Table S5 also shows a diverse temperature effect depending on the Pluronic and SAIL nature. 29 Commonly, the temperature promotes micelle fusion processes increasing the sizes. In 30 Pluronic/[ $\left.\mathrm{P}_{4,4,4,14}\right] \mathrm{Cl}$ mixtures at their CPTs (see Table S2), reverse Pluronics, 10R5/[P $\left.4,4,4,14\right] \mathrm{Cl}$ $31\left(76^{\circ} \mathrm{C}\right)$ and $31 \mathrm{R} 1 /\left[\mathrm{P}_{4,4,4,14}\right] \mathrm{Cl}\left(27^{\circ} \mathrm{C}\right)$, showed bigger micelles but the size was practically unaffected 
1 in normal Pluronics, L-35/[P $4,4,4,14] \mathrm{Cl}\left(95^{\circ} \mathrm{C}\right)$ and $\mathrm{L}-31 /\left[\mathrm{P}_{4,4,4,14}\right] \mathrm{Cl}\left(47^{\circ} \mathrm{C}\right)$. However, the

2 imidazolium exhibited a different behaviour with the $10 \mathrm{R} 5 /\left[\mathrm{C}_{14} \mathrm{mim}\right] \mathrm{Cl}\left(88^{\circ} \mathrm{C}\right)$ displaying smaller

3 micelles whilst bigger ones were obtained in the $\mathrm{L} 35 /\left[\mathrm{C}_{14} \mathrm{mim}\right] \mathrm{Cl}\left(95^{\circ} \mathrm{C}\right)$. Conversely, for low

4 PEG:PPG Pluronic ratios, the same number of micelles and $\mathrm{N}_{\mathrm{a}}$, were maintained, but the micelle

5 diameter was increased. Table S6 shows a summary of the $\mathrm{N}_{\mathrm{a}}$ and micelle diameter $(\varnothing)$ evolution

6 when CPT is attained in each system.

7 The diverse trend exhibited above could be explained by the fact that the Pluronic/SAIL 8 micellar growth with temperature increase may depend on whether the SAIL presents an LCST or 9 UCST behaviour. Although the micelle size might not increase for an LCST system by fusion, 10 dehydration of the interface could allow for the formation or larger aggregates by coalescence.

11 This is similar to what we observed in our previous work with the $\left[\mathrm{P}_{4,4,4,14}\right] \mathrm{Cl}$ system. ${ }^{58} \mathrm{~A}$ 12 surfactant presenting an UCST behaviour, such as imidazolium ILs, can be considered more as a 13 traditional salt, for which we would expect a temperature increase to have less effect on the micelle 14 size. In addition, mixtures of Pluronic and SAILs are likely to exhibit an even more complex 15 behaviour compared with individual solutions of Pluronics and SAILs, and it is difficult to 16 extrapolate results based on a limited number of micelles to the complex phenomena of phase 17 separation.

18 Finally, it can be seen that the effect of SAIL on the cloud point is somewhat more pronounced 19 for the normal Pluronic L-35 than for the reverse Pluronic 10R5. Taking into account that both 20 polymers have the same composition, only differing in the arrangement of the PPG and PEG 21 blocks, suggests that the differences are purely related to surface effects. However, the effects of 22 the different SAIL cations on the micelle surface structure are also quite similar for these two 23 Pluronics (compare Figures S9 and S10). The most probable explanation, therefore, lies in the 24 ability of 10R5 to form cross-links between micelles during self-assembly. As discussed in our 25 previous publication, ${ }^{7}$ this ability is responsible for the measured differences in the cloud points 26 of the two Pluronics in aqueous solutions without SAIL. When SAIL is added to the mixture, the 27 10R5 chains still retain their ability to cross-link in the very initial stages of the micelle formation 28 as shown in Figure S12. The initial cross-link aggregates, which only appear at the beginning, can 29 contribute to counteract the effect of electrostatic repulsion induced by the SAIL. This 30 circumstance facilitates the micelles to be closer to each other, despite the fact that in the final 31 equilibrium state, the micelles are separated. As a consequence, the SAIL effect is more 
1 pronounced in the normal L-35 Pluronic, where cross-links are absent, and the system is dominated

2 by electrostatics.

4. Conclusions

5 This work provides a detailed study on the impact of SAILs on the cloud points of a series of 6 Pluronic copolymers with different characteristics, namely normal $v s$ reverse, and with different 7 PEG content. In all cases, it was observed that the increase in the system hydrophobicity led to a 8 decrease in the cloud point temperature as a result of an intricate balance of hydrophobic forces 9 and inter micellar interactions. The more pronounced cloud point increase of the mixed 10 imidazolium-based systems was a result of the unscreened imidazolium charge in comparison with

11 the screened phosphonium head group. A weak alkyl-chain length effect was found in the micelle 12 distribution of mixtures of Pluronics with $\left[\mathrm{C}_{10} \mathrm{mim}\right] \mathrm{Cl}$ and $\left[\mathrm{C}_{14} \mathrm{mim}\right] \mathrm{Cl}$. However, a more detailed 13 study including larger imidazolium and phosphonium alkyl-chain lengths is needed to shed further 14 light into this issue The bulkier alkyl side chains of $\left[\mathrm{P}_{4,4,4,14}\right] \mathrm{Cl}$ screen the SAIL charge and displace 15 it towards the inside of the Pluronic micelle core, reducing the impact of electrostatic repulsion 16 between neighbouring micelles and thus, favouring their coalescence. The chain length of the 17 imidazolium SAIL had virtually no effect on the cloud point of Pluronics with small cores and 18 large coronas, since even small cations were able to induce inter micellar repulsions. In contrast, 19 for Pluronics with larger cores, SAIL cations with larger chain lengths are needed to induce such 20 effects. Our results suggest that there may be a minimum cation chain length for each type of 21 Pluronic, below which the cloud point is not affected. More systematic studies on Pluronic/SAIL 22 mixtures with controlled sizes of hydrophobic groups are needed to confirm this assertion. The 23 overall impact of SAILs of the Pluronic micellar aqueous solutions addressed in this study was a 24 reduction in the micelle fusion events, increasing the number of aggregates compared with the 25 Pluronic aqueous solution references whilst the micelle size is increased by the presence of 26 adsorbed SAIL moieties. Although the measures described above give us confidence that the 27 results we are reporting correspond to thermodynamic equilibrium, we cannot exclude the 28 possibility of kinetic effects that are longer than the time scale of our simulations.

29 The remarkably simple and transferable model herein proposed herein was able to capture the 30 subtle differences in the behaviour of four distinct Pluronics in aqueous solution over a range of 31 concentrations from dilute to mildly concentrated ${ }^{7}$ as well as the interaction between dilute 
1 solutions of SAILs and Pluronics. This represents a significant advancement in both simulation

2 and understanding of triblock copolymer aggregation. Experimental results, validated by the

3 simulations, indicate that the LCST transition for triblock copolymers can be varied by the careful

4 adjustment of the PEG:PPG ratio and the micelle surface characteristic, i.e. normal $v s$ reverse

5 Pluronics, as previously reported. ${ }^{7}$ This can be further tuned by addition of small amounts of

6 SAILs. The nature of the SAIL and concentration as well as the SAIL-Pluronic interactions were

7 found to strongly influence the cloud point of the polymer. Ortona et al. ${ }^{25}$ suggested that the

8 Pluronic-surfactant interactions are driven by a combination of several factors, in which some of

9 them can be distinct from the known surfactant-surfactant interactions. Surface functionalization

10 of the polymeric micelle by addition of an electrostatic component with different strength

11 (imidazolium $v s$ phosphonium-based SAILs) is of significant interest for several fields. SAILs

12 were shown to extract a number of high value inorganic and organic compounds. This work offers

13 a predictive model to study different copolymers while elucidating the fundamental thermal

14 response of these systems, which is required for the cloud point extraction of distinct compounds.

15 Furthermore, this work provides, for the first time, an intuitive computer simulation framework to

16 study mixtures of Pluronics and SAILs, opening the door for designing tailor-made thermal

17 controlled solvents by computer simulations. 


\section{Supporting Information}

2 The Supporting Information is available free of charge on the ACS Publications website at 3 DOI:XXX.

4 Characteristics of the Pluronic systems used in this study, the PPG and PEG content, \% in PEG 5 and molecular weight as well as a description of all simulation runs carried out at $25^{\circ} \mathrm{C}$ and CPT.

6 Experimental binodal CPT curves for all Pluronic aqueous solutions and the SAIL mixtures up to

$718 \mathrm{wt} \%$ of Pluronic concentration. Detailed description of the initial stages of micelle formation 8 of L-35 and L-31 and their mixtures with $\left[\mathrm{C}_{14} \mathrm{mim}\right] \mathrm{Cl}$ and $\left[\mathrm{P}_{4,4,4,14}\right] \mathrm{Cl}$ at $25^{\circ} \mathrm{C}$. A detailed picture 9 of the initial stages of $\mathrm{L}-35$ with $\left[\mathrm{C}_{14} \mathrm{mim}\right] \mathrm{Cl}$ and $\left[\mathrm{P}_{4,4,4,14}\right] \mathrm{Cl}$ mixtures at $25^{\circ} \mathrm{C}$ with $0.3 \mathrm{wt} \%$ of 10 SAIL concentration where L-35, SAIL monomers and hybrid L-35/SAIL aggregates are shown. 11 Micelle density profiles of 10R5, L-35 and 31R1 aqueous solutions and after addition of $12\left[\mathrm{C}_{10} \mathrm{mim}\right] \mathrm{Cl},\left[\mathrm{C}_{14} \mathrm{mim}\right] \mathrm{Cl}$ and $\left[\mathrm{P}_{4,4,4,14}\right] \mathrm{Cl}$. A detailed picture of the initial stages of the 10R5 + $13\left[\mathrm{C}_{14} \mathrm{mim}\right] \mathrm{Cl}$ micelle formation at the CPT highlighting cross-linked formation.

\section{Acknowledgements}

16 This work was developed within the scope of the project CICECO-Aveiro Institute of Materials, 17 UIDB/50011/2020 \& UIDP/50011/2020, financed by national funds through the FCT/MEC and 18 when appropriate co-financed by FEDER under the PT2020 Partnership Agreement. The authors 19 are also grateful for the national fund through the Portuguese Foundation for Science and 20 Technology (FCT) for the doctoral grant SFRH/BD/101683/2014 of F.A. Vicente. German Perez21 Sanchez and Nicolas Schaeffer acknowledge the national funds (OE), through FCT - Fundação 22 para a Ciência e a Tecnologia, I.P., in the scope of the framework contract foreseen in the numbers 23 4, 5 and 6 of the article 23, of the Decree-Law 57/2016, of August 29, changed by Law 57/2017, 24 of July 19. 
References

2 (1) Bodratti, A.; Alexandridis, P. Formulation of Poloxamers for Drug Delivery. J. Funct. Biomater. 2018, 9 (1), 11.

(2) Wang, Y.; Hu, X.; Han, J.; Ni, L.; Tang, X.; Hu, Y.; Chen, T. Integrated Method of

Thermosensitive Triblock Copolymer-Salt Aqueous Two Phase Extraction and Dialysis Membrane Separation for Purification of Lycium Barbarum Polysaccharide. Food Chem. 2016, 194, 257-264.

(3) de Lemos, L. R.; Campos, R. A.; Rodrigues, G. D.; da Silva, L. H. M.; da Silva, M. C. H. Green Separation of Copper and Zinc Using Triblock Copolymer Aqueous Two-Phase Systems. Sep. Purif. Technol. 2013, 115, 107-113.

(4) Wanka, G.; Hoffmann, H.; Ulbricht, W. Phase Diagrams and Aggregation Behavior of Poly (Oxyethylene)-Poly (Oxypropylene)-Poly (Oxyethylene) Triblock Copolymers in Aqueous Solutions. Macromolecules 1994, 27 (15), 4145-4159.

(5) Mortensen, K.; Brown, W.; Joergensen, E. Phase Behavior of Poly(Propylene Oxide)Poly(Ethylene Oxide)-Poly(Propylene Oxide) Triblock Copolymer Melt and Aqueous Solutions. Macromolecules 1994, 27 (20), 5654-5666.

(6) Tsui, H.-W.; Wang, J.-H.; Hsu, Y.-H.; Chen, L.-J. Study of Heat of Micellization and Phase Separation for Pluronic Aqueous Solutions by Using a High Sensitivity Differential Scanning Calorimetry. Colloid Polym. Sci. 2010, 288 (18), 1687-1696.

Pérez-Sánchez, G.; Vicente, F. A.; Schaeffer, N.; Cardoso, I. S.; Ventura, S. P. M.; Jorge, M.; Coutinho, J. A. P. Rationalizing the Phase Behavior of Triblock Copolymers through Experiments and Molecular Simulations. J. Phys. Chem. C 2019, 123 (34), 21224-21236.

(8) Soares, R. R. G.; Azevedo, A. M.; Van Alstine, J. M.; Aires-Barros, M. R. Partitioning in Aqueous Two-Phase Systems: Analysis of Strengths, Weaknesses, Opportunities and Threats. Biotechnol. J. 2015, 10 (8), 1158-1169.

Blesic, M.; Marques, M. H.; Plechkova, N. V; Seddon, K. R.; Rebelo, L. P. N.; Lopes, A. Self-Aggregation of Ionic Liquids: Micelle Formation in Aqueous Solution. Green Chem. 
2007, 9 (5), 481-490.

(10) Dong, B.; Li, N.; Zheng, L.; Yu, L.; Inoue, T. Surface Adsorption and Micelle Formation of Surface Active Ionic Liquids in Aqueous Solution. Langmuir 2007, 23 (8), 4178-4182.

(11) Smirnova, N. a; Vanin, A. a; Safonova, E. a; Pukinsky, I. B.; Anufrikov, Y. a; Makarov, A. L. Self-Assembly in Aqueous Solutions of Imidazolium Ionic Liquids and Their Mixtures with an Anionic Surfactant. J. Colloid Interface Sci. 2009, 336 (2), 793-802.

(12) Behera, K.; Pandey, S. Interaction between Ionic Liquid and Zwitterionic Surfactant: A Comparative Study of Two Ionic Liquids with Different Anions. J. Colloid Interface Sci. 2009, 331 (1), 196-205.

(13) Singh, K.; Marangoni, D. G.; Quinn, J. G.; Singer, R. D. Spontaneous Vesicle Formation with an Ionic Liquid Amphiphile. J. Colloid Interface Sci. 2009, 335 (1), 105-111.

(14) Pramanik, R.; Sarkar, S.; Ghatak, C.; Rao, V. G.; Mandal, S.; Sarkar, N. Effects of 1-Butyl3-Methyl Imidazolium Tetrafluoroborate Ionic Liquid on Triton X-100 Aqueous Micelles: Solvent and Rotational Relaxation Studies. J. Phys. Chem. B 2011, 115 (21), 6957-6963.

(15) Chen, L. G.; Bermudez, H. Solubility and Aggregation of Charged Surfactants in Ionic Liquids. Langmuir 2012, 28 (2), 1157-1162.

(16) Chen, L. G.; Bermudez, H. Charge Screening between Anionic and Cationic Surfactants in Ionic Liquids. Langmuir 2013, 29 (9), 2805-2808.

(17) Miskolczy, Z.; Sebők-Nagy, K.; Biczók, L.; Göktürk, S. Aggregation and Micelle Formation of Ionic Liquids in Aqueous Solution. Chem. Phys. Lett. 2004, 400 (4-6), 296300.

(18) Umapathi, R.; Venkatesu, P. Thermo-Responsive Triblock Copolymer Phase Transition Behaviour in Imidazolium-Based Ionic Liquids: Role of the Effect of Alkyl Chain Length of Cations. J. Colloid Interface Sci. 2017, 485, 183-191.

(19) Madhusudhana Reddy, P.; Venkatesu, P. Influence of Ionic Liquids on the Critical Micellization Temperature of a Tri-Block Co-Polymer in Aqueous Media. J. Colloid 
Interface Sci. 2014, 420, 166-173.

(20) Singh, D. K.; Sastry, N. V; Trivedi, P. A. Amphiphilic Copolymers and Surface Active Ionic Liquid Systems in Aqueous Media - Surface Active and Aggregation Characteristics. Colloids Surfaces A Physicochem. Eng. Asp. 2017, 524, 111-126.

(21) Phani Kumar, B. V. N.; Reddy, R. R.; Pan, A.; Aswal, V. K.; Tsuchiya, K.; Prameela, G. K. S.; Abe, M.; Mandal, A. B.; Moulik, S. P. Physicochemical Understanding of SelfAggregation and Microstructure of a Surface-Active Ionic Liquid [C4mim] [C8OSO3] Mixed with a Reverse Pluronic 10R5 (PO8EO22PO8). ACS Omega 2018, 3 (5), 5155-5164.

(22) Zheng, L.; Guo, C.; Wang, J.; Liang, X.; Chen, S.; Ma, J.; Yang, B.; Jiang, Y.; Liu, H. Effect of Ionic Liquids on the Aggregation Behavior of PEO-PPO-PEO Block Copolymers in Aqueous Solution. J. Phys. Chem. B 2007, 111 (6), 1327-1333.

(23) Dai, S.; Tam, K. C.; Li, L. Isothermal Titration Calorimetric Studies on Interactions of Ionic Surfactant and Poly(Oxypropylene)-Poly(Oxyethylene)- Poly(Oxypropylene) Triblock Copolymers in Aqueous Solutions. Macromolecules 2001, 34 (20), 7049-7055.

(24) Mata, J.; Joshi, T.; Varade, D.; Ghosh, G.; Bahadur, P. Aggregation Behavior of a PEOPPO-PEO Block Copolymer + Ionic Surfactants Mixed Systems in Water and Aqueous Salt Solutions. Colloids Surfaces A Physicochem. Eng. Asp. 2004, 247 (1-3), 1-7.

(25) Ortona, O.; D’Errico, G.; Paduano, L.; Vitagliano, V. Interaction between Cationic, Anionic, and Non-Ionic Surfactants with ABA Block Copolymer Pluronic PE6200 and with BAB Reverse Block Copolymer Pluronic 25R4. J. Colloid Interface Sci. 2006, 301 (1), $63-$ 77.

(26) Sharma, R.; Kang, T. S.; Mahajan, R. K. Complexation of Triblock Reverse Copolymer 10R5 with Surface Active Ionic Liquids in Aqueous Medium: A Physico-Chemical Study. RSC Adv. 2015, 5 (21), 16349-16360.

(27) Liu, X.; Hu, J.; Huang, Y.; Fang, Y. Aggregation Behavior of Surface Active Dialkylimidazolium Ionic Liquids $[\mathrm{C} 12 \mathrm{Cnim}] \mathrm{Br}(\mathrm{n}=1-4)$ in Aqueous Solutions. $J$. Surfactants Deterg. 2013, 16 (4), 539-546. 
1 (28) Lunagariya, J.; Kumar, N. S.; Asif, M.; Dhar, A.; Vekariya, R. L. Dependency of Anion and

Chain Length of Imidazolium Based Ionic Liquid on Micellization of the Block Copolymer F127 in Aqueous Solution: An Experimental Deep Insight. Polymers (Basel). 2017, 9 (7), 285.

(29) Khan, I.; Umapathi, R.; Neves, M. C.; Coutinho, J. A. P.; Venkatesu, P. Structural Insights into the Effect of Cholinium-Based Ionic Liquids on the Critical Micellization Temperature of Aqueous Triblock Copolymers. Phys. Chem. Chem. Phys. 2016, 18 (12), 8342-8351.

(30) Vicente, F. A.; Malpiedi, L. P.; Silva, F. A. e; Jr., A. P.; Coutinho, J. A. P.; Ventura, S. P. M. Design of Novel Aqueous Micellar Two-Phase Systems Using Ionic Liquids as CoSurfactants for the Selective Extraction of (Bio)Molecules. Sep. Purif. Technol. 2014, 135, 259-267.

(31) Vicente, F. A.; Cardoso, I. S.; Sintra, T. E.; Lemus, J.; Marques, E. F.; Ventura, S. P. M.; Coutinho, J. A. P. Impact of Surface Active Ionic Liquids on the Cloud Points of Nonionic Surfactants and the Formation of Aqueous Micellar Two-Phase Systems. J. Phys. Chem. B 2017, 121 (37), 8742-8755.

(32) Schaeffer, N.; Passos, H.; Gras, M.; Mogilireddy, V.; Leal, J. P.; Perez-Sanchez, G.; Gomes, J. R. B.; Billard, I.; Papaiconomou, N.; Coutinho, J. A. P. Mechanism of Ionic-Liquid-Based Acidic Aqueous Biphasic System Formation. Phys. Chem. Chem. Phys. 2018.

(33) Crespo, E. A.; Schaeffer, N.; Coutinho, J. A. P.; Perez-Sanchez, G. Improved Coarse-Grain Model to Unravel the Phase Behavior of 1-Alkyl-3-Methylimidazolium-Based Ionic Liquids through Molecular Dynamics Simulations. J. Colloid Interface Sci. 2020, 574, 324336.

(34) Marrink, S. J.; Risselada, H. J.; Yefimov, S.; Tieleman, D. P.; de Vries, A. H. The MARTINI Force Field: Coarse Grained Model for Biomolecular Simulations. J. Phys. Chem. B 2007, 111 (27), 7812-7824.

(35) Alessandri, R.; Uusitalo, J. J.; De Vries, A. H.; Havenith, R. W. A.; Marrink, S. J. Bulk Heterojunction Morphologies with Atomistic Resolution from Coarse-Grain Solvent Evaporation Simulations. J. Am. Chem. Soc. 2017, 139 (10), 3697-3705. 
1 (36) Vazquez-Salazar, L. I.; Selle, M.; de Vries, A. H.; Marrink, S.-J.; Souza, P. C. T. Martini Coarse-Grained Models of Imidazolium-Based Ionic Liquids: From Nanostructural Organization to Liquid-Liquid Extraction. 2020.

(37) Panizon, E.; Bochicchio, D.; Monticelli, L.; Rossi, G. MARTINI Coarse-Grained Models of Polyethylene and Polypropylene. J. Phys. Chem. B 2015, 119 (25), 8209-8216.

(38) Yesylevskyy, S. O.; Schäfer, L. V.; Sengupta, D.; Marrink, S. J. Polarizable Water Model

(39) Bedrov, D.; Borodin, O.; Li, Z.; Smith, G. D. Influence of Polarization on Structural, Thermodynamic, and Dynamic Properties of Ionic Liquids Obtained from Molecular Dynamics Simulations. J. Phys. Chem. B 2010, 114 (15), 4984-4997.

(40) Michalowsky, J.; Schäfer, L. V.; Holm, C.; Smiatek, J. A Refined Polarizable Water Model

for the Coarse-Grained MARTINI Force Field. PLoS Comput. Biol. 2010, 6 (6), e1000810. for the Coarse-Grained MARTINI Force Field with Long-Range Electrostatic Interactions. J. Chem. Phys. 2017, 146 (5), 054501.

(41) Abraham, M. J.; Murtola, T.; Schulz, R.; Páll, S.; Smith, J. C.; Hess, B.; Lindahl, E. GROMACS: High Performance Molecular Simulations through Multi-Level Parallelism from Laptops to Supercomputers. SoftwareX 2015, 1-2, 19-25.

(42) Hockney, R. .; Goel, S. .; Eastwood, J. . Quiet High-Resolution Computer Models of a Plasma. J. Comput. Phys. 1974, 14 (2), 148-158.

(43) Verlet, L. Computer "Experiments" on Classical Fluids. I. Thermodynamical Properties of Lennard-Jones Molecules. Phys. Rev. 1967, 159 (1), 98-103.

(44) Darden, T.; York, D.; Pedersen, L. Particle Mesh Ewald: An N·log(N) Method for Ewald Sums in Large Systems. J. Chem. Phys. 1993, 98 (12), 10089-10092.

(45) Hess, B.; Bekker, H.; Berendsen, H. J. C.; Fraaije, J. G. E. M. LINCS: A Linear Constraint Solver for Molecular Simulations. J. Comput. Chem. 1997, 18 (12), 1463-1472.

(46) Bussi, G.; Donadio, D.; Parrinello, M. Canonical Sampling through Velocity Rescaling. $J$. Chem. Phys. 2007, 126 (1), 014101. 
1 (47) Hoover, W. G. Canonical Dynamics: Equilibrium Phase-Space Distributions. Phys. Rev. A 1985, 31 (3), 1695-1697.

(48) Nosé, S. A Unified Formulation of the Constant Temperature Molecular Dynamics Methods. J. Chem. Phys. 1984, 81 (1), 511-519.

(49) Parrinello, M.; Rahman, A. Polymorphic Transitions in Single Crystals: A New Molecular Dynamics Method. J. Appl. Phys. 1981, 52 (12), 7182-7190.

(50) Humphrey, W.; Dalke, A.; Schulten, K. VMD: Visual Molecular Dynamics. J. Mol. Graph. 1996, 14 (1), 33-38.

(51) Jorge, M. Molecular Dynamics Simulation of Self-Assembly of $\mathrm{n}$ Decyltrimethylammonium Bromide Micelles. Langmuir 2008, 24 (11), 5714-5725.

(52) Pérez-Sánchez, G.; Gomes, J. R. B.; Jorge, M. Modeling Self-Assembly of Silica/Surfactant Mesostructures in the Templated Synthesis of Nanoporous Solids. Langmuir 2013, 29 (7).

(53) Hoshen, J.; Kopelman, R. Percolation and Cluster Distribution. I. Cluster Multiple Labeling Technique and Critical Concentration Algorithm. Phys. Rev. B 1976, 14 (8), 3438-3445.

(54) Pérez-Sánchez, G.; Vicente, F. A.; Schaeffer, N.; Cardoso, I. S.; Ventura, S. P. M.; Jorge, M.; Coutinho, J. A. P. Rationalizing the Phase Behavior of Triblock Copolymers through Experiments and Molecular Simulations. J. Phys. Chem. C 2019, 123 (34), 21224-21236.

(55) Blesic, M.; Marques, M. H.; Plechkova, N. V; Seddon, K. R.; Rebelo, L. P. N.; Lopes, A. Self-Aggregation of Ionic Liquids: Micelle Formation in Aqueous Solution. Green Chem. 2007, 9 (5), 481-490.

(56) Torres, F. A. E.; de Almeida Francisco, A. C.; Pereira, J. F. B.; Santos-Ebinuma, V. de C. Imidazolium-Based Ionic Liquids as Co-Surfactants in Aqueous Micellar Two-Phase Systems Composed of Nonionic Surfactants and Their Aptitude for Recovery of Natural Colorants from Fermented Broth. Sep. Purif. Technol. 2018, 196, 262-269.

(57) Alexandridis, P.; Alan Hatton, T. Poly(Ethylene Oxide) $\square$ poly(Propylene Oxide) $\square$ poly(Ethylene Oxide) Block Copolymer Surfactants in Aqueous Solutions and at 
Interfaces: Thermodynamics, Structure, Dynamics, and Modeling. Colloids Surfaces A Physicochem. Eng. Asp. 1995, 96 (1-2), 1-46.

3 (58) Schaeffer, N.; Pérez-Sánchez, G.; Passos, H.; Gomes, J. R. B.; Papaiconomou, N.; Coutinho, J. A. P. Mechanisms of Phase Separation in Temperature-Responsive Acidic Aqueous Biphasic Systems. Phys. Chem. Chem. Phys. 2019, 21 (14), 7462-7473.

\section{TOC Graphic}

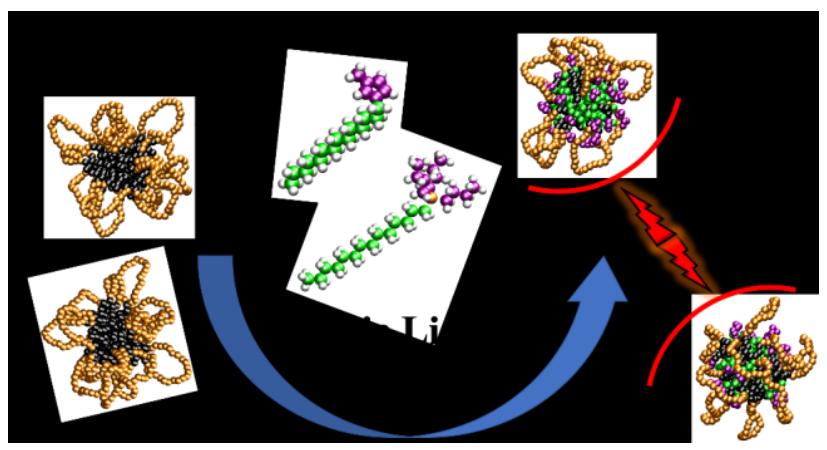




\section{Supporting Information}

\section{Unravelling the Interactions between Surface-}

Active Ionic Liquids and Triblock-Copolymers for the Design of Thermal Responsive Systems

Germán Pérez-Sánchez ${ }^{1,+, *}$, Filipa A. Vicente ${ }^{1,+}$, Nicolas Schaeffer $^{l}$, Inês S. Cardoso ${ }^{1}$, Sónia P. M. Ventura ${ }^{1}$, Miguel Jorge $e^{2, *}$ and João A. P. Coutinho ${ }^{1}$

${ }^{1}$ CICECO, Departamento de Química, Universidade de Aveiro, 3810-193 Aveiro, Portugal

${ }^{2}$ Department of Chemical and Process Engineering, University of Strathclyde, 75 Montrose Street, Glasgow G1 1XJ, United Kingdom

*Corresponding authors

${ }^{\dagger}$ Both authors contributed equally

Campus Universitário de Santiago, Universidade de Aveiro, Aveiro, Portugal

Tel: +351-234-370200; Fax: +351-234-370084; E-mail addresses: gperez@ua.pt, miguel.jorge@strath.ac.uk. 


\section{Contents:}

Table S1. Description of the Pluronics used in this study. PPG and PEG content, \% in PEG and molecular weight data are provided.

Tables S2-S3. Detailed description of the Pluronic/SAIL mixtures simulated in this work.

Table S4. Number of micelles $(\mathrm{N})$, Pluronic aggregation number $\left(\mathrm{N}_{\mathrm{a}}\right)$ and averaged micelle diameter $(\varnothing)$ obtained after $3 \mu$ s of simulation time for the 10R5 aqueous solution and their SAIL mixtures including the double sized (60)10R5/Water simulation to evaluate any system-size effects.

Table S5. Number of micelles $(\mathrm{N})$, Pluronic aggregation number $\left(\mathrm{N}_{\mathrm{a}}\right)$ and averaged micelle diameter $(\varnothing)$ obtained after $3 \mu$ s of simulation time for the all Pluronic aqueous solutions and their SAIL mixtures at $298 \mathrm{~K}$ and their CPT.

Table S6. Pluronic aggregation number $\left(\mathrm{N}_{\mathrm{a}}\right)$ and averaged micelle diameter $(\varnothing)$ evolution when temperature is raised to their CPTs taking the $25^{\circ} \mathrm{C}$ to values as a reference.

Figure S1. Binodal curves of different Pluronic concentrations with $0.3 \mathrm{wt} \%$ of SAIL + $0.18 \mathrm{M}$ of McIlvaine buffer $\mathrm{pH} 7.0$ obtained experimentally.

Figure S2. Micelle density profile comparison for $\left[\mathrm{P}_{4,4,4,14}\right] \mathrm{Cl}$ under regular and polarizable MARTINI water model.

Figure S3. E $E_{\text {tot }}$ profile over $3 \mu$ s of simulation time for 10R5, L-35, 31R1 and L-31 aqueous solutions and their mixtures with $\left[\mathrm{C}_{144 \mathrm{mim}}\right] \mathrm{Cl}$ and $\left[\mathrm{P}_{4,4,4,14}\right] \mathrm{Cl}$ at $298 \mathrm{~K}$.

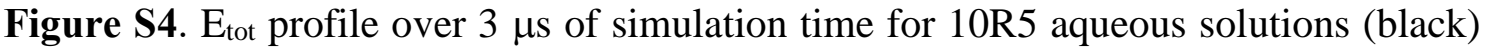
and mixtures with $\left[\mathrm{C}_{14} \mathrm{mim}\right] \mathrm{Cl}$ (yellow) and $\left[\mathrm{P}_{4,4,4,14}\right] \mathrm{Cl}$ (purple). 
Figure S5. Micelle aggregation number (Na) along $3000 \mathrm{~ns}$ of simulation time obtained with the cluster counting code at $25^{\circ} \mathrm{C}$.

Figure S6. Detailed description of the initial stages of micelle formation obtained from the molecular dynamics simulations of L-35 in aqueous solutions and their mixtures with $\left[\mathrm{C}_{14} \mathrm{mim}\right] \mathrm{Cl}$ and $\left[\mathrm{P}_{4,4,4,14}\right] \mathrm{Cl}$.

Figure S7. Detailed picture of the initial stages in the MD simulations of L-35, demonstrating the $\left[\mathrm{C}_{14} \mathrm{mim}\right] \mathrm{Cl}$ and $\left[\mathrm{P}_{4,4,4,14}\right] \mathrm{Cl}$ SAIL micelle formation in parallel with Pluronic/SAIL micelles.

Figure S8. Detailed description of the initial stages of micelle formation obtained from the molecular dynamics simulations of L-31 in aqueous solutions and their mixtures with $\left[\mathrm{C}_{14} \mathrm{mim}\right] \mathrm{Cl}$ and $\left[\mathrm{P}_{4,4,4,14}\right] \mathrm{Cl}$.

Figures S9-S11. Micelle density profiles for 10R5 (S5), L-35 (S6) and 31R1 (S7) Pluronics without SAILs and after addition of $\left[\mathrm{C}_{10 \mathrm{mim}}\right] \mathrm{Cl},\left[\mathrm{C}_{14 \mathrm{mim}}\right] \mathrm{Cl}$ and $\left[\mathrm{P}_{4,4,4,14}\right] \mathrm{Cl}$.

Figure S12. Simulation MD snapshot displaying a detailed scenario of the initial stages of the $10 \mathrm{R} 5+\left[\mathrm{C}_{14} \mathrm{mim}\right] \mathrm{Cl}$ micelle formation. 
Table S1. Pluronic systems used in this study: two normal copolymers, L-31 and L-35, and two reverse ones, 31R1 and 10R5. The colour code denotes the apolar PPG in black and the polar PEG in orange. The $\%$ PEG is based on the molecular weight of each coarse-grain bead, 58 and 44 AMU for PPG and PEG, respectively.

\begin{tabular}{|c|c|c|c|c|c|}
\hline Pluronic & $\% \mathrm{PEG}$ & $\mathrm{M}_{\mathrm{w}}$ & \#PEG & \#PPG & \#PEC \\
\hline L-31 & 16 & 1100 & 2 & 16 & 2 \\
\hline L-35 & 53 & 1900 & 11 & 16 & 11 \\
\hline & & & \#PPG & \#PEG & \#PPG \\
\hline 31R1 & 11 & 3300 & 26 & 8 & 26 \\
\hline 10R5 & 49 & 2000 & 8 & 22 & 8 \\
\hline
\end{tabular}


Table S2. Molecular dynamics simulations carried out for 10R5, L-35, L-31 and 31R1 Pluronics and their SAIL mixtures. All systems contain 30 Pluronic chains, the SAIL is the number of ionic liquid pairs and $\mathrm{W}$ is the number of CG water beads (each corresponding to 4 real water molecules). The temperature was fixed to the experimental cloud point temperature values obtained in the laboratory ( $c f$. Figure S1).

\begin{tabular}{|c|c|c|c|}
\hline Systems & SAIL & $\mathbf{W}$ & Temperature $\left({ }^{\circ} \mathrm{C}\right)$ \\
\hline 10R5 $1 w t \%$ & 0 & 80000 & 61 \\
\hline 10R5 $1 w t \%\left[\mathrm{C}_{10} \mathrm{mim}\right] \mathrm{Cl} 0.3 \mathrm{wt} \%$ & 68 & 80000 & 72 \\
\hline 10R5 $1 w t \%\left[\mathrm{C}_{14} \mathrm{mim}\right] \mathrm{Cl} 0.3 \mathrm{wt} \%$ & 56 & 80000 & 88 \\
\hline 10R5 $1 \mathrm{wt} \%\left[\mathrm{P}_{4,4,4,14}\right] \mathrm{Cl} 0.3 \mathrm{wt} \%$ & 40 & 80000 & 76 \\
\hline $\mathrm{L}-351 w t \%$ & 0 & 78000 & 61 \\
\hline $\mathrm{L}-351 \mathrm{wt} \%\left[\mathrm{C}_{10} \mathrm{mim}\right] \mathrm{Cl} 0.3 \mathrm{wt} \%$ & 66 & 78000 & 92 \\
\hline $\mathrm{L}-351 \mathrm{wt} \%\left[\mathrm{C}_{14} \mathrm{mim}\right] \mathrm{Cl} 0.3 \mathrm{wt} \%$ & 55 & 78000 & 95 \\
\hline $\mathrm{L}-351 w t \%\left[\mathrm{P}_{4,4,4,14}\right] \mathrm{Cl} 0.3 \mathrm{wt} \%$ & 39 & 78000 & 95 \\
\hline L-31 $1 w t \%$ & 0 & 45000 & 35 \\
\hline $\mathrm{L}-311 \mathrm{wt} \%\left[\mathrm{C}_{10} \mathrm{mim}\right] \mathrm{Cl} 0.3 \mathrm{wt} \%$ & 38 & 45000 & 33 \\
\hline $\mathrm{L}-311 \mathrm{wt} \%\left[\mathrm{C}_{14} \mathrm{mim}\right] \mathrm{Cl} 0.3 \mathrm{wt} \%$ & 31 & 45000 & 49 \\
\hline $\mathrm{L}-311 w t \%\left[\mathrm{P}_{4,4,4,14}\right] \mathrm{Cl} 0.3 \mathrm{wt} \%$ & 22 & 45000 & 47 \\
\hline 31R1 $1 w t \%$ & 0 & 135000 & 30 \\
\hline $31 \mathrm{R} 11 w t \%\left[\mathrm{C}_{10} \mathrm{mim}\right] \mathrm{Cl} 0.3 \mathrm{wt} \%$ & 115 & 135000 & 22 \\
\hline 31R1 $1 w t \%\left[\mathrm{C}_{14} \mathrm{mim}\right] \mathrm{Cl} 0.3 \mathrm{wt} \%$ & 94 & 135000 & 40 \\
\hline 31R1 $1 w t \%\left[\mathrm{P}_{4,4,4,14}\right] \mathrm{Cl} 0.3 \mathrm{wt} \%$ & 68 & 135000 & 27 \\
\hline
\end{tabular}

Table S3. Molecular dynamics simulations for 10R5, 31R1, L-35 and L-31, and their SAIL mixtures at $25^{\circ} \mathrm{C}$. All systems contain 30 Pluronic chains, the SAIL is the number of ionic liquid pairs with $\mathrm{W}$ the number of CG water beads (each corresponding to 4 real water molecules).

\begin{tabular}{|c|c|c|}
\hline Systems & SAIL & $\mathbf{W}$ \\
\hline 10R5 $1 w t \%$ & 0 & 80000 \\
\hline 10R5 $1 \mathrm{wt} \%\left[\mathrm{C}_{14} \mathrm{mim}\right] \mathrm{Cl} 0.3 \mathrm{wt} \%$ & 56 & 80000 \\
\hline 10R5 $1 \mathrm{wt} \%\left[\mathrm{P}_{4,4,4,14}\right] \mathrm{Cl} 0.3 \mathrm{wt} \%$ & 40 & 80000 \\
\hline $\mathrm{L}-351 w t \%$ & 0 & 78000 \\
\hline L-35 $1 w t \%\left[\mathrm{C}_{14} \mathrm{mim}\right] \mathrm{Cl} 0.3 \mathrm{wt} \%$ & 56 & 78000 \\
\hline $\mathrm{L}-351 w t \%\left[\mathrm{P}_{4,4,4,14}\right] \mathrm{Cl} 0.3 \mathrm{wt} \%$ & 40 & 78000 \\
\hline L-31 $1 w t \%$ & 0 & 45000 \\
\hline $\mathrm{L}-311 \mathrm{wt} \%\left[\mathrm{C}_{14} \mathrm{mim}\right] \mathrm{Cl} 0.3 \mathrm{wt} \%$ & 32 & 45000 \\
\hline $\mathrm{L}-311 w t \%\left[\mathrm{P}_{4,4,4,14}\right] \mathrm{Cl} 0.3 \mathrm{wt} \%$ & 23 & 45000 \\
\hline 31R1 $1 w t \%$ & 0 & 135000 \\
\hline $31 \mathrm{R} 11 \mathrm{wt} \%\left[\mathrm{C}_{14} \mathrm{mim}\right] \mathrm{Cl} 0.3 \mathrm{wt} \%$ & 94 & 135000 \\
\hline 31R1 $1 w t \%\left[\mathrm{P}_{4,4,4,14}\right] \mathrm{Cl} 0.3 \mathrm{wt} \%$ & 68 & 135000 \\
\hline
\end{tabular}


Table S4. Number of micelles $(\mathrm{N})$, Pluronic aggregation number $\left(\mathrm{N}_{\mathrm{a}}\right)$ and averaged micelle diameter $(\varnothing)$ obtained after $3 \mu$ s of simulation time for the 10R5 aqueous solution and their SAIL mixtures. The double sized (60)10R5/Water simulation was extended to $9 \mu$ s of simulation time. The values between brackets denote the number of Pluronic moieties. The last $1 \mu$ s of simulation time was used to obtain the data.

\begin{tabular}{cccccccccc} 
& \multicolumn{3}{c}{$(30) 10 \mathrm{R} 5$} & \multicolumn{3}{c}{$(60) 10 \mathrm{R} 5$} & \multicolumn{2}{c}{$(60) 10 \mathrm{R} 59 \mu \mathrm{s}$} \\
\cline { 2 - 9 } & $\mathrm{N}$ & $\mathrm{N}_{\mathrm{a}}$ & $\varnothing(\mathrm{nm})$ & $\mathrm{N}$ & $\mathrm{N}_{\mathrm{a}}$ & $\varnothing(\mathrm{nm})$ & $\mathrm{N}$ & $\mathrm{N}_{\mathrm{a}}$ & $\varnothing(\mathrm{nm})$ \\
\hline NoSAIL & 3 & 10 & 3.4 & 7 & 8.6 & 3.0 & 3 & 10 & 3.3 \\
\hline$\left[\mathrm{C}_{14 \mathrm{mim}}\right] \mathrm{Cl}$ & 3 & 10 & 4.2 & 6 & 10 & 4.0 & - & - & - \\
\hline$\left[\mathrm{P}_{4,4,4,14}\right] \mathrm{Cl}$ & 3 & 10 & 4.4 & 6 & 10 & 4.2 & - & - & - \\
\hline
\end{tabular}

Table S5. Number of micelles $(\mathrm{N})$, Pluronic aggregation number $\left(\mathrm{N}_{\mathrm{a}}\right)$ and averaged micelle diameter $(\varnothing)$ obtained after $3 \mu$ s of simulation time for the all Pluronic aqueous solutions and their SAIL mixtures at $25^{\circ} \mathrm{C}$ and at the CPT.

\begin{tabular}{cccccccccccc}
\hline & 10R5 & \multicolumn{1}{c}{ L-35 } & \multicolumn{1}{c}{$31 \mathrm{R} 1$} & \multicolumn{3}{c}{ L-31 } \\
\hline $\mathrm{N}$ & $\mathrm{N}_{\mathrm{a}}$ & $\begin{array}{c}\varnothing \\
(\mathrm{nm})\end{array}$ & $\mathrm{N}$ & $\mathrm{N}_{\mathrm{a}}$ & $\begin{array}{c}\varnothing \\
(\mathrm{nm})\end{array}$ & $\mathrm{N}$ & $\mathrm{N}_{\mathrm{a}}$ & $\begin{array}{c}\varnothing \\
(\mathrm{nm})\end{array}$ & $\mathrm{N}$ & $\mathrm{N}_{\mathrm{a}}$ & $\begin{array}{c}\varnothing \\
(\mathrm{nm})\end{array}$ \\
\hline
\end{tabular}

\begin{tabular}{ccccccccccccc}
\hline $25^{\circ} \mathrm{c}$ & & & & & & & & & & & & \\
\hline NoSAIL & 3 & 10 & 3.4 & 4 & 7.5 & 3.1 & 1 & 30 & 5.1 & 1 & 30 & 3.9 \\
\hline$\left[\mathrm{C}_{14} \mathrm{mim}\right] \mathrm{Cl}$ & 3 & 10 & 4.2 & 6 & 5 & 3.0 & 3 & 10 & 4.5 & 1 & 30 & 4.1 \\
\hline$\left[\mathrm{P}_{4,4,4,14}\right] \mathrm{Cl}$ & 3 & 10 & 4.4 & 3 & 10 & 4.2 & 3 & 10 & 5.0 & 1 & 30 & 4.4 \\
\hline $\mathrm{CPT}$ & & & & & & & & & & & & \\
\hline NoSAIL & 2 & 15 & 3.6 & 3 & 10 & 3.7 & 1 & 30 & 5.1 & 1 & 30 & 3.9 \\
\hline$\left[\mathrm{C}_{10} \mathrm{mim}\right] \mathrm{Cl}$ & 4 & 7.5 & 3.6 & 4 & 7 & 3.3 & 4 & 7.5 & 4.1 & 1 & 30 & 4.2 \\
\hline$\left[\mathrm{C}_{14} \mathrm{mim}\right] \mathrm{Cl}$ & 4 & 7.5 & 3.4 & 4 & 7.5 & 3.7 & 3 & 10 & 6.0 & 1 & 30 & 4.4 \\
\hline$\left[\mathrm{P}_{4,4,4,14}\right] \mathrm{Cl}$ & 1 & 30 & 5.7 & 3 & 10 & 4.1 & 2 & 15 & 5.2 & 1 & 30 & 4.6 \\
\hline
\end{tabular}

Table S6. Pluronic aggregation number $\left(\mathrm{N}_{\mathrm{a}}\right)$ and averaged micelle diameter $(\varnothing)$ evolution when temperature is raised to their CPTs taking the $25^{\circ} \mathrm{C}$ to values as a reference.

\begin{tabular}{cccccccc}
\hline \multicolumn{2}{c}{ 10R5 } & \multicolumn{2}{c}{ L-35 } & \multicolumn{2}{c}{ 31R1 } & L-31 & \\
\hline $\mathrm{N}_{\mathrm{a}}$ & $\varnothing$ & $\mathrm{N}_{\mathrm{a}}$ & $\varnothing$ & $\mathrm{N}_{\mathrm{a}}$ & $\varnothing$ & $\mathrm{N}_{\mathrm{a}}$ & $\varnothing$ \\
\hline Bigger & Bigger & Bigger & Bigger & Same & Same & Same & Same \\
\hline Smaller & Smaller & Bigger & Bigger & Same & Bigger & Same & Bigger \\
\hline Bigger & Bigger & Same & Same & Bigger & Bigger & Same & Bigger \\
\hline
\end{tabular}



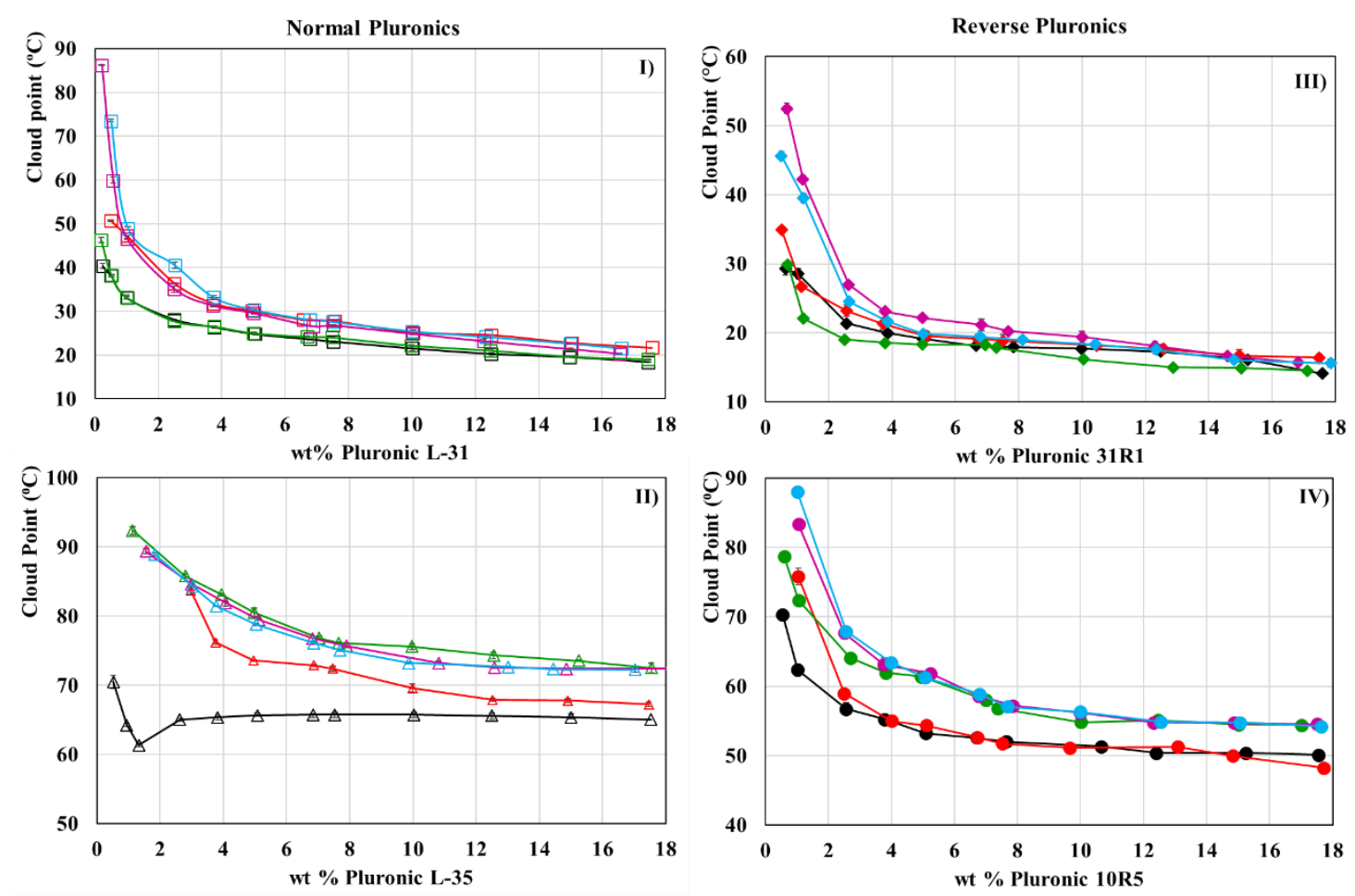

Figure S1. Binodal curves of Pluronic copolymers $+0.3 \mathrm{wt} \%$ of SAIL $+0.18 \mathrm{M}$ of McIlvaine buffer pH 7.0. I) $\square$, Pluronic L-31. II) $\Delta$, Pluronic L-35. III) •, Pluronic 31R1. IV) •, Pluronic 10R5. The SAILs added to the AMTPS are:,$-\left[\mathrm{C}_{10} \mathrm{mim}\right] \mathrm{Cl} ;-,\left[\mathrm{C}_{12} \mathrm{mim}\right] \mathrm{Cl} ;-,\left[\mathrm{C}_{14} \mathrm{mim}\right] \mathrm{Cl} ;-,\left[\mathrm{P}_{4,4,4,14}\right] \mathrm{Cl}$. The black line corresponds to the reference system without SAIL.

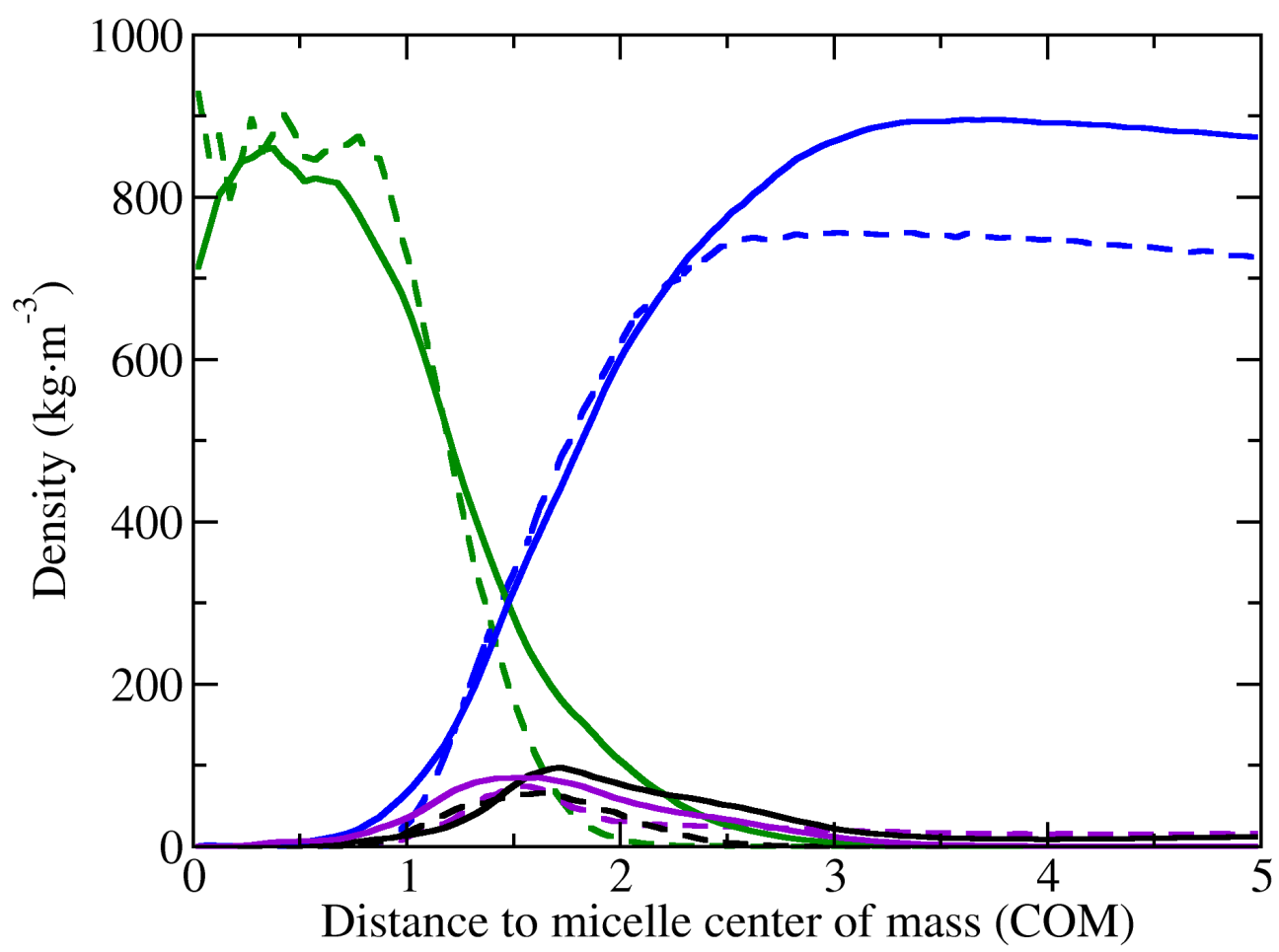

Figure S2. Micelle density profile comparison for $\left[\mathrm{P}_{4,4,4,14}\right] \mathrm{Cl}$ under regular and polarizable MARTINI water model. The colour code is as follows: $\left[\mathrm{P}_{4,4,4,14}\right]^{+}$alkyl-chain and head groups in green and purple, 
respectively. $\mathrm{Cl}^{-}$anions are in black and water in blue. The dashed purple lines show the profile for the polarizable water system whilst solid lines represent the regular water model system used in the manuscript.
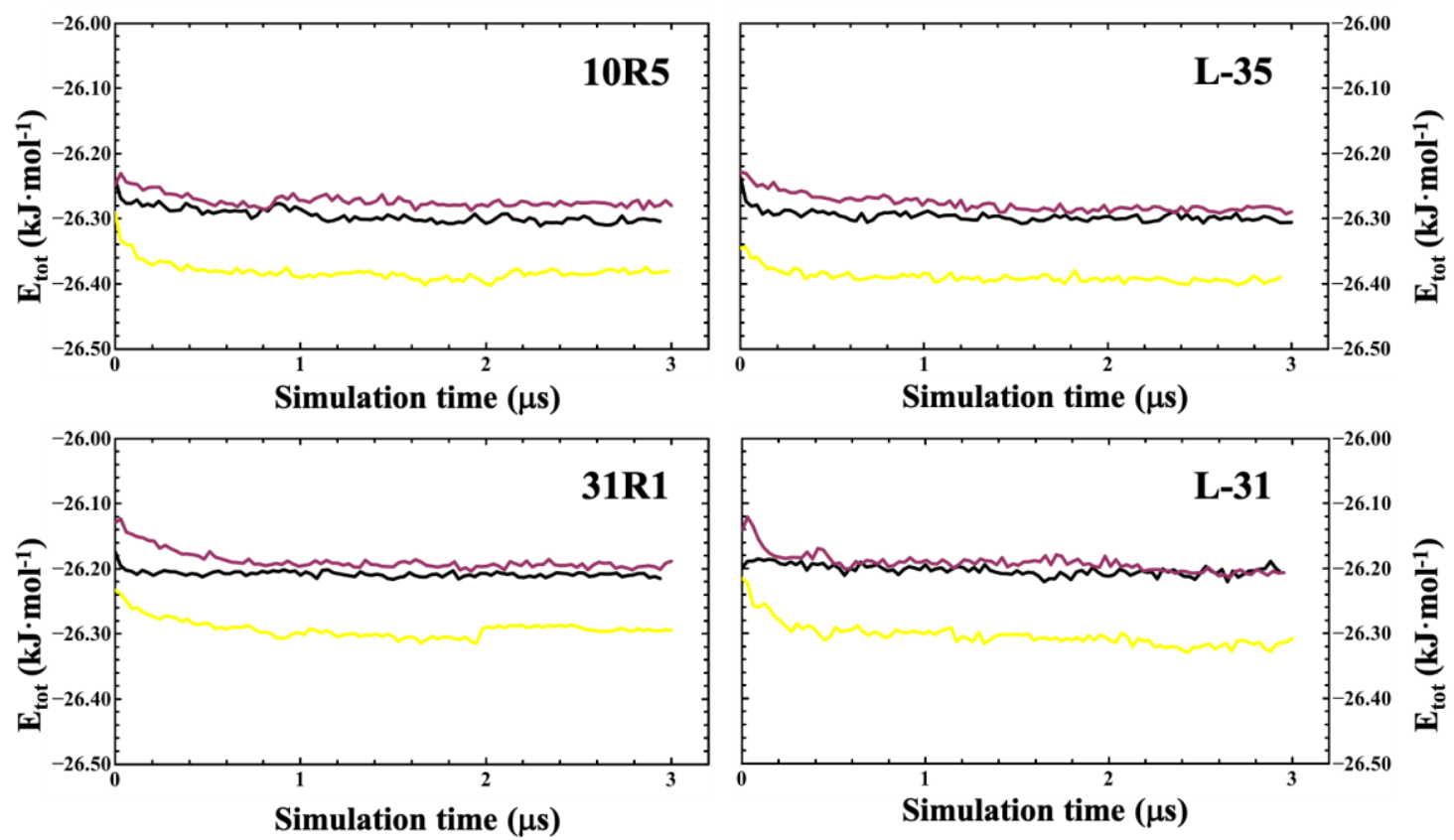

Figure S3. $\mathrm{E}_{\text {tot }}$ profile over $3 \mu$ s of simulation time for 10R5, L-35, 31R1 and L-31 aqueous solutions (black) and their mixtures with $\left[\mathrm{C}_{14} \mathrm{mim}\right] \mathrm{Cl}$ (yellow) and $\left[\mathrm{P}_{4,4,4,14}\right] \mathrm{Cl}$ (purple) at $298 \mathrm{~K}$.

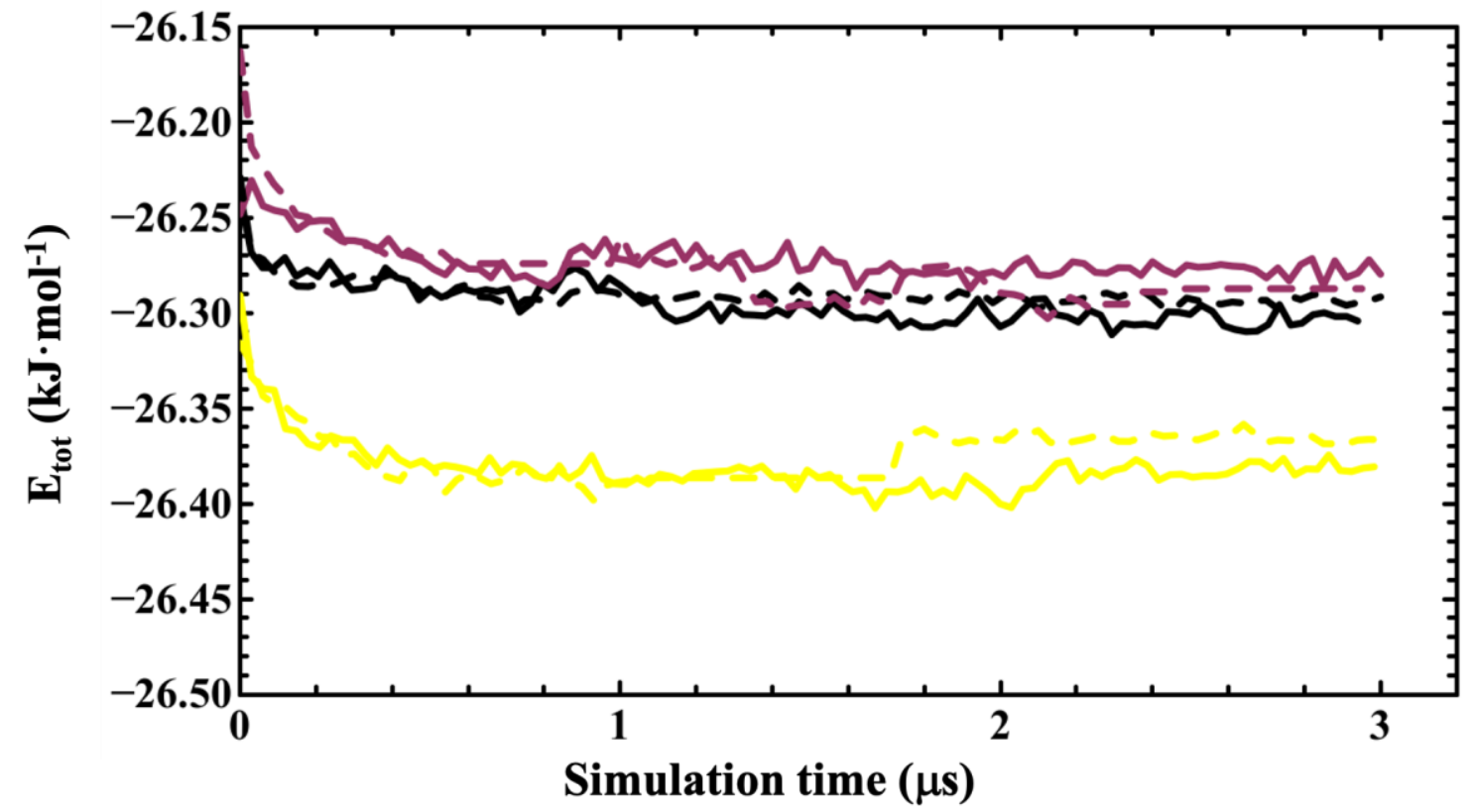


Figure S4. $E_{\text {tot }}$ profile over $3 \mu$ s of simulation time for 10R5 aqueous solutions (black) and mixtures with $\left[\mathrm{C}_{14} \mathrm{mim}\right] \mathrm{Cl}$ (yellow) and $\left[\mathrm{P}_{4,4,4,14}\right] \mathrm{Cl}$ (purple). The solid lines are for systems with 30 Pluronics and the dashed lines for systems with 60 Pluronics.
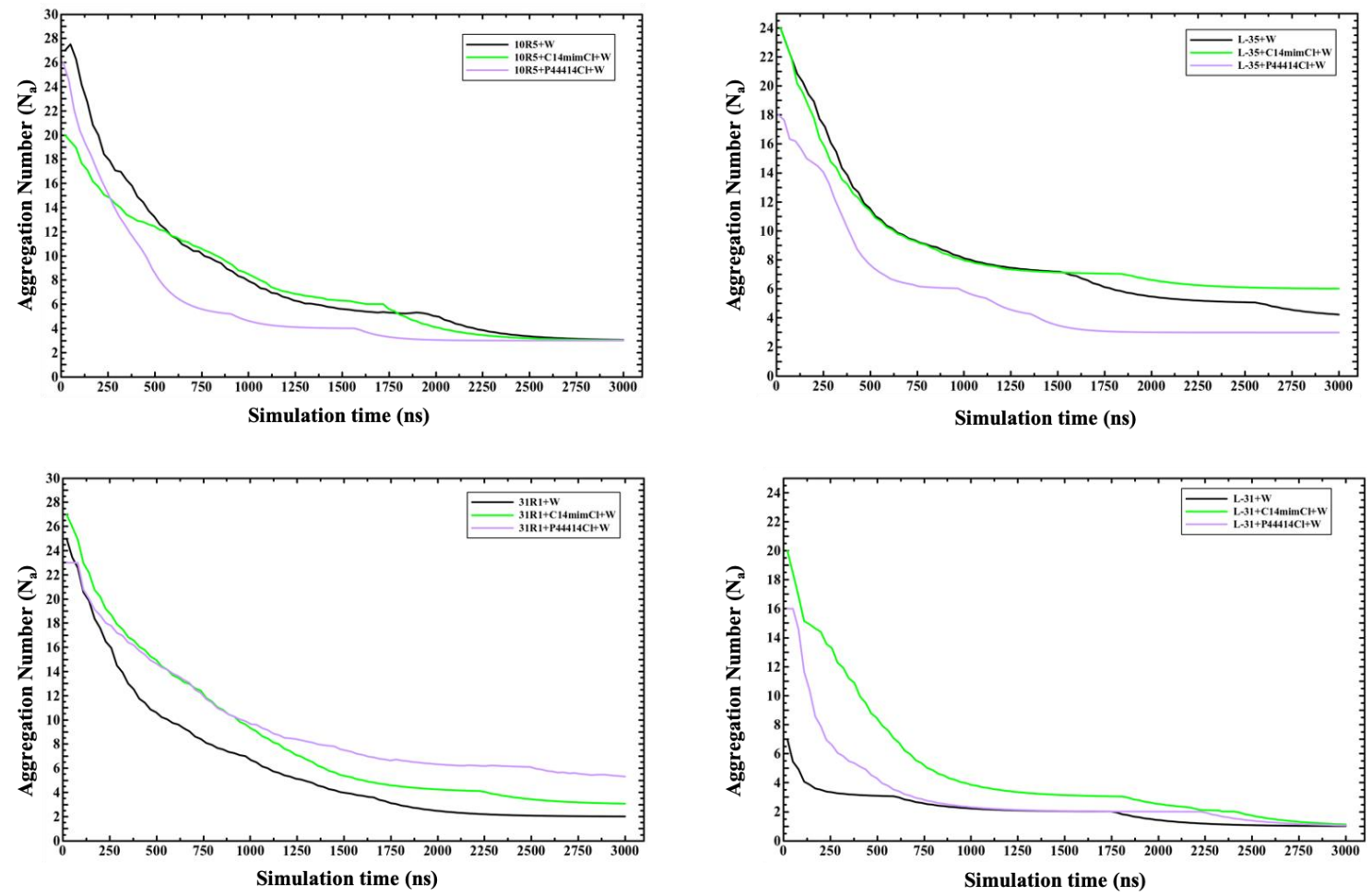

Figure S5. Micelle aggregation number $\left(\mathrm{N}_{\mathrm{a}}\right)$ along $3000 \mathrm{~ns}$ of simulation time obtained with the cluster counting code at $25^{\circ} \mathrm{C}$. Pluronic aqueous solutions are in black and their SAIL mixtures with $\left[\mathrm{C}_{14} \mathrm{mim}\right] \mathrm{Cl}$ and $\left[\mathrm{P}_{4,4,4,14}\right] \mathrm{Cl}$ are in green and purple, respectively. 


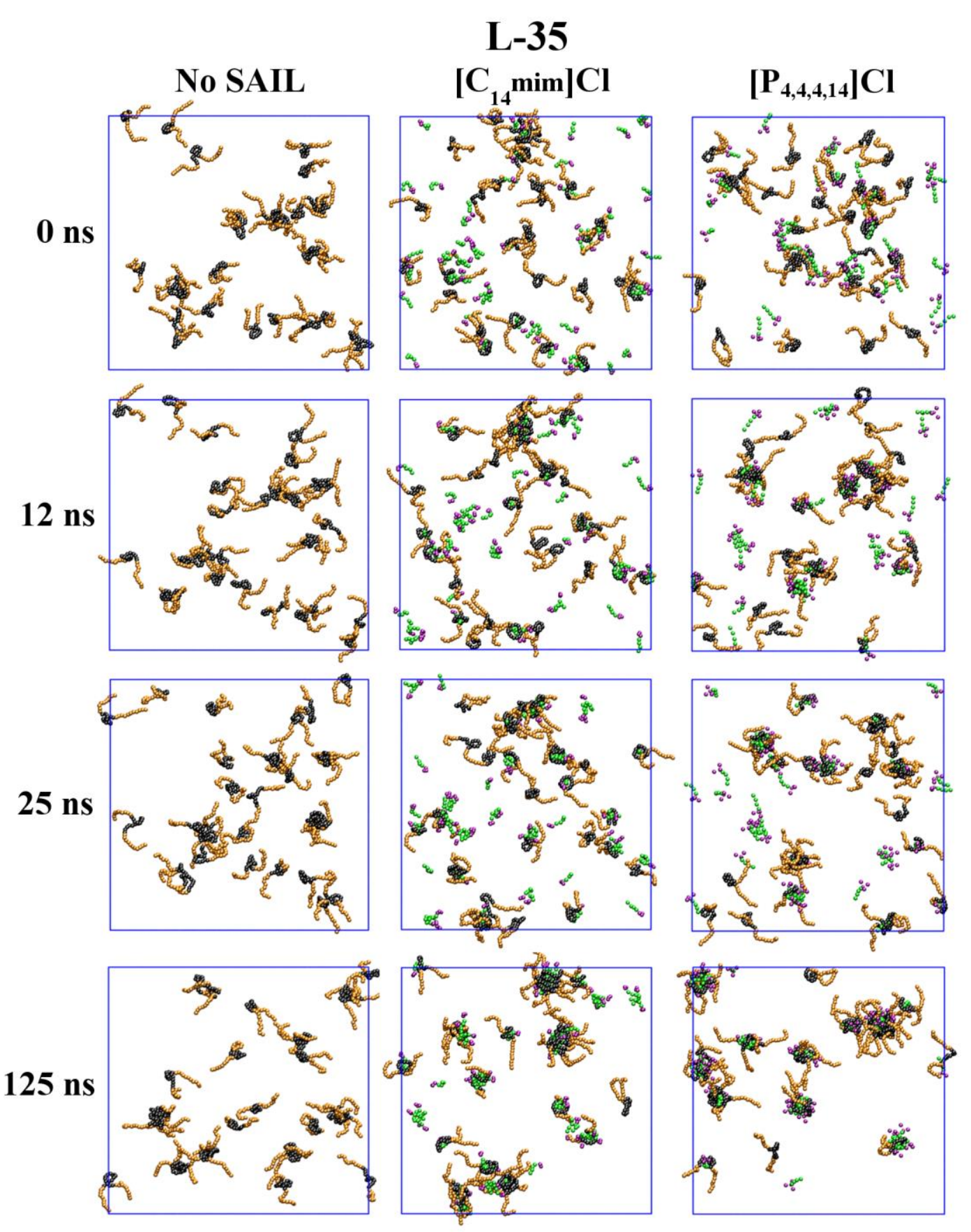

Figure S6. Detailed description of the initial stages of micelle formation in L-35 aqueous solutions (left column) and their mixtures with $\left[\mathrm{C}_{14} \mathrm{mim}\right] \mathrm{Cl}$ (middle column) and $\left[\mathrm{P}_{4,4,4,14}\right] \mathrm{Cl}$ (right column). The temperature was fixed to $25^{\circ} \mathrm{C}$. The colour code is as follows: for Pluronics, the PPG and PEG are in black and orange, respectively; for SAILs, the hydrophobic alkyl-chain tail is green whereas the imidazolium ring in $\left[\mathrm{C}_{\mathrm{n}} \mathrm{mim}\right] \mathrm{Cl}$ and the butyl head groups in $\left[\mathrm{P}_{4,4,4,14}\right] \mathrm{Cl}$ are coloured in purple. Water and chloride ions were removed for clarity. 


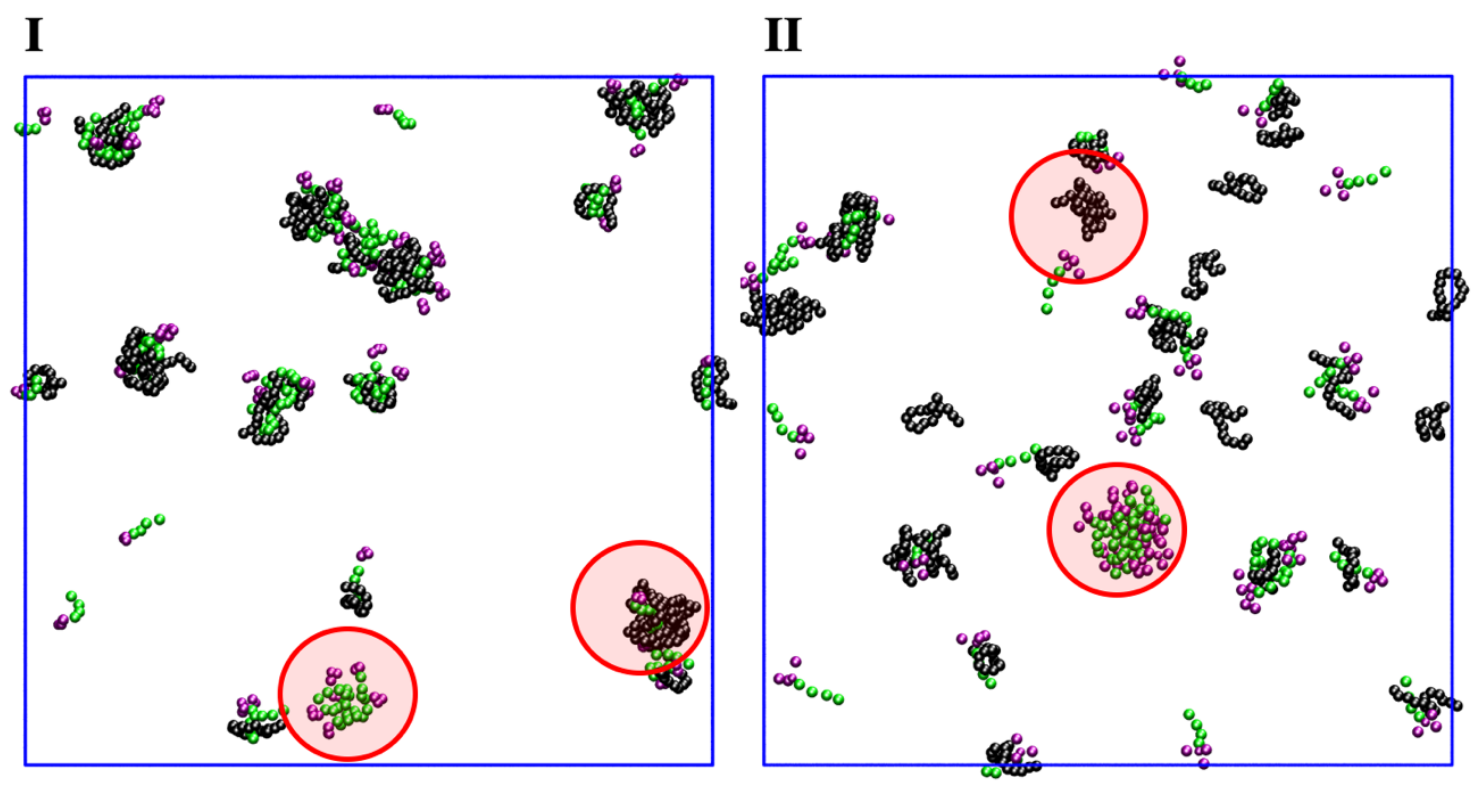

Figure S7. Detailed picture of the initial stages of SAIL and L-35 micelle formation at 0.3 wt $\%$ of I) $\left[\mathrm{C}_{14} \mathrm{mim}\right] \mathrm{Cl}$ and II) $\left[\mathrm{P}_{4,4,4,14}\right] \mathrm{Cl}$. Some L-35 and SAIL micelles are discernible surrounded by red circles in the initial aggregation stages. The colour code is PPG in black, SAIL alkyl-chains in green, and SAIL headgroups in purple. The PEG in L-35, the chloride ions and water molecules were removed for clarity. 


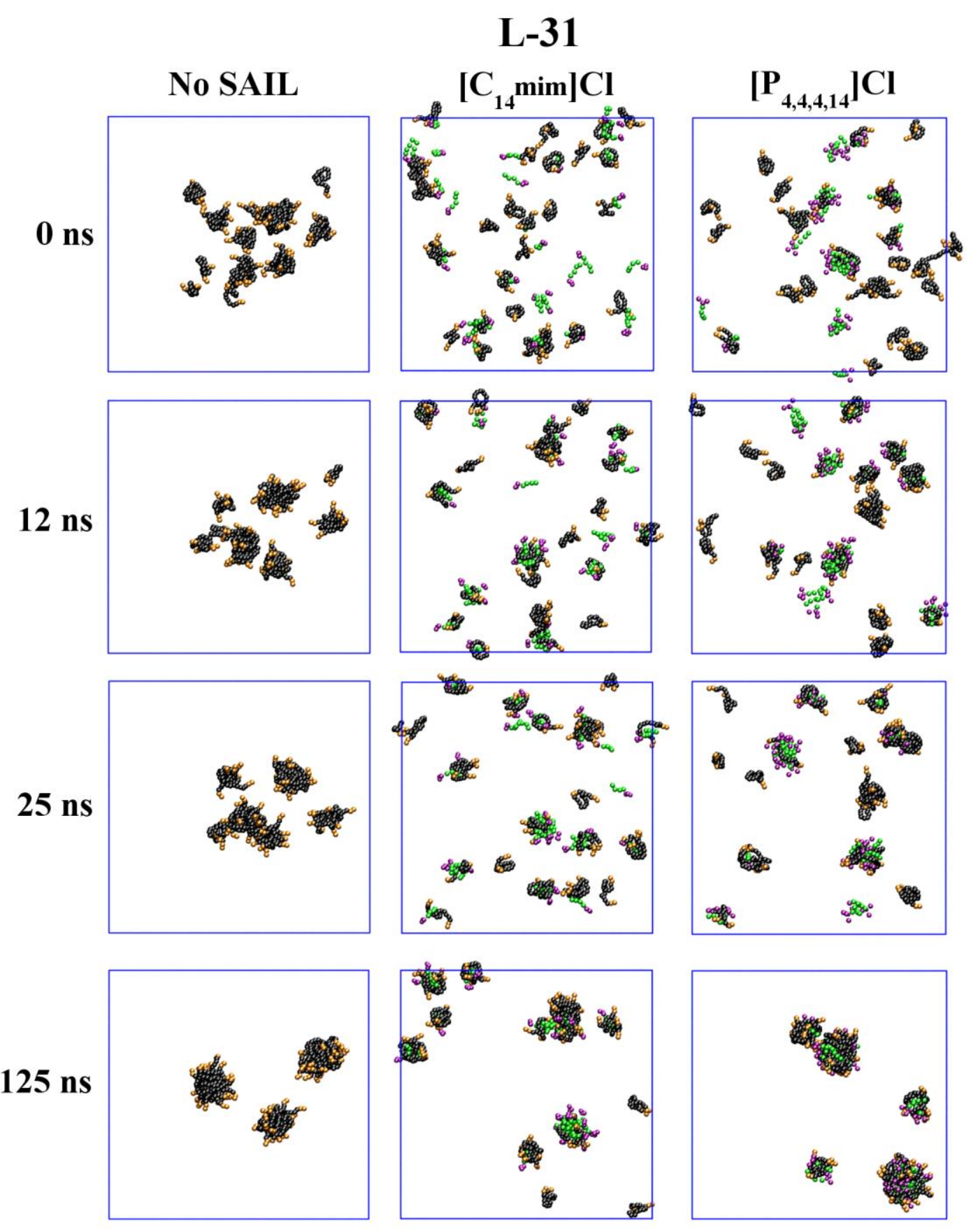

Figure S8. Detailed description of the initial stages of micelle formation in L-31 aqueous solutions (left column) and their mixtures with $\left[\mathrm{C}_{14} \mathrm{mim}\right] \mathrm{Cl}$ (middle column) and $\left[\mathrm{P}_{4,4,4,14}\right] \mathrm{Cl}$ (right column). The temperature was fixed to $25^{\circ} \mathrm{C}$. The colour code is the same as Figure $\mathbf{S 2}$. 

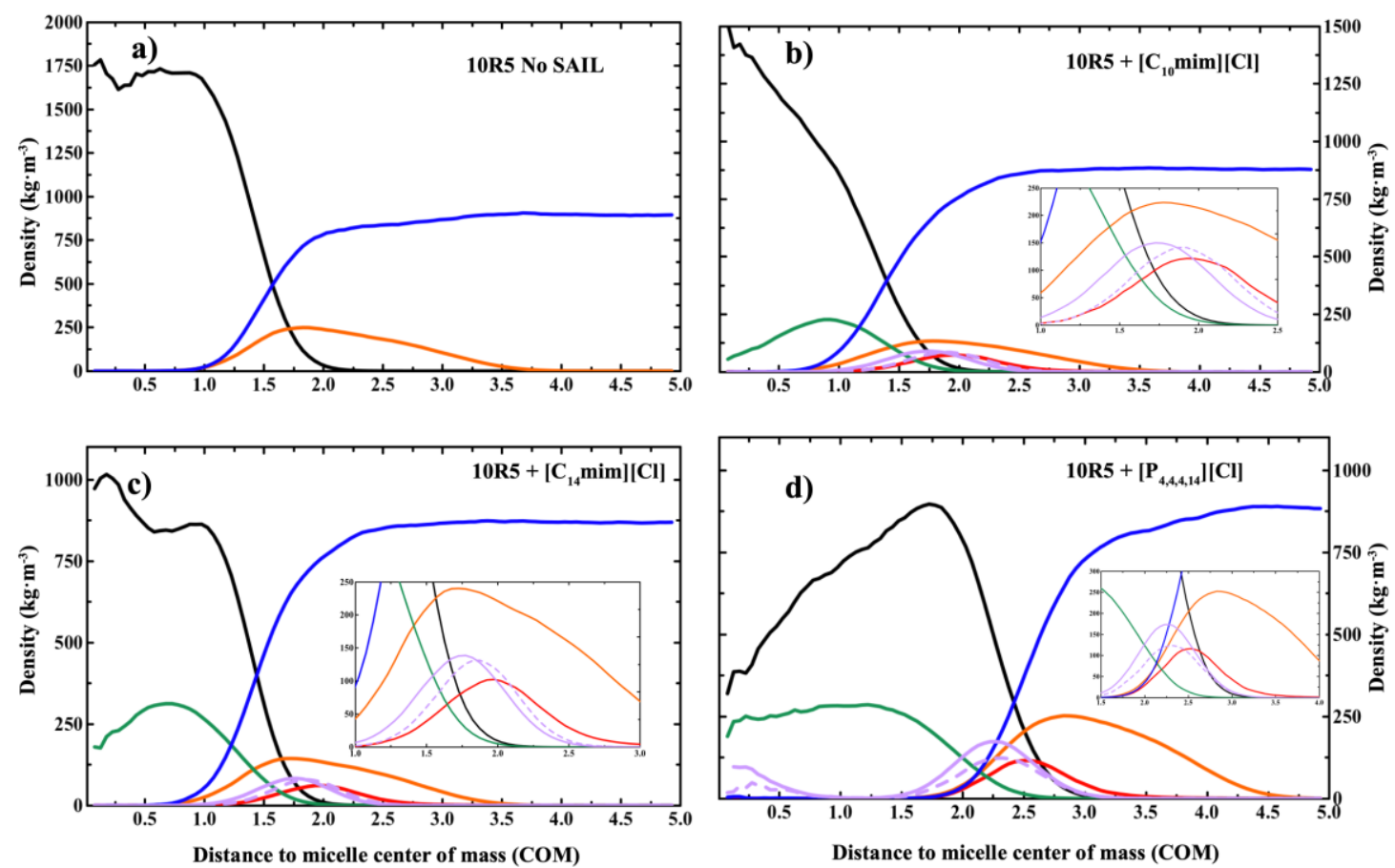

Figure S9. Micelle density profiles for 10R5 solutions without SAILs (a) and after addition of $\left[\mathrm{C}_{10} \mathrm{mim}\right] \mathrm{Cl}(\mathrm{b}),\left[\mathrm{C}_{14} \mathrm{mim}\right] \mathrm{Cl}(\mathrm{c})$ and $\left[\mathrm{P}_{4,4,4,14}\right] \mathrm{Cl}(\mathrm{d})$. The colour code is as follows: SAIL alkyl-chain tails in green, SAIL head groups in purple, chlorides in red, water in blue, PEG micelle corona in orange and PPG micelle hydrophobic core in black. The dashed purple line shows the profile for the charged site of the SAIL head group, multiplied by a factor of 3 for ease of visualization. The insets show a blow up of the head-group region for the systems with SAIL. 

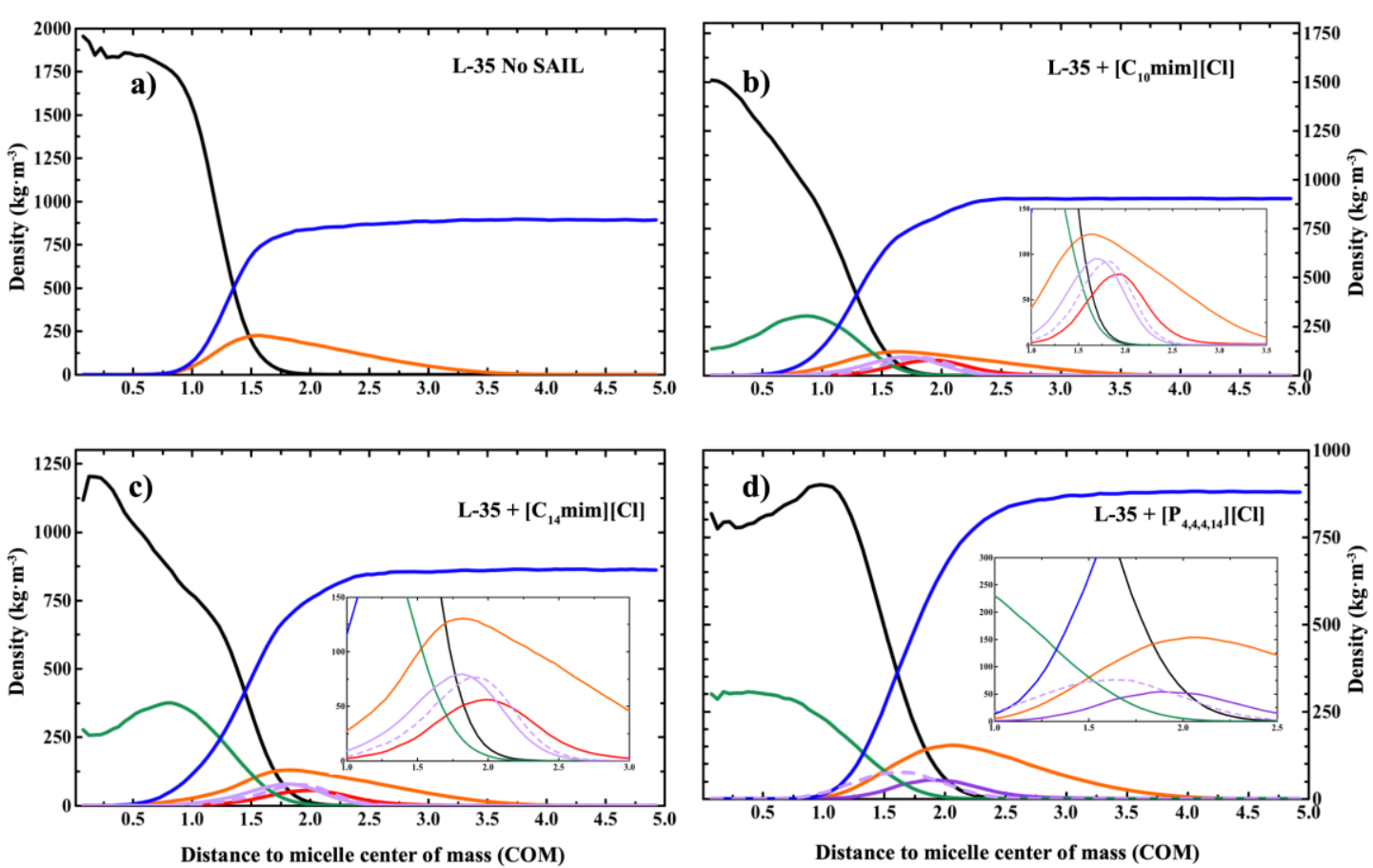

Figure S10. Micelle density profiles for L-35 solutions without SAILs (a) and after addition of $\left[\mathrm{C}_{10} \mathrm{mim}\right] \mathrm{Cl}$ (b), $\left[\mathrm{C}_{14} \mathrm{mim}\right] \mathrm{Cl}(\mathrm{c})$ and $\left[\mathrm{P}_{4,4,4,14}\right] \mathrm{Cl}(\mathrm{d})$. The colour code is the same as in Figure $\mathbf{S 9}$. The insets show a blow up of the head-group region for the systems with SAIL. 

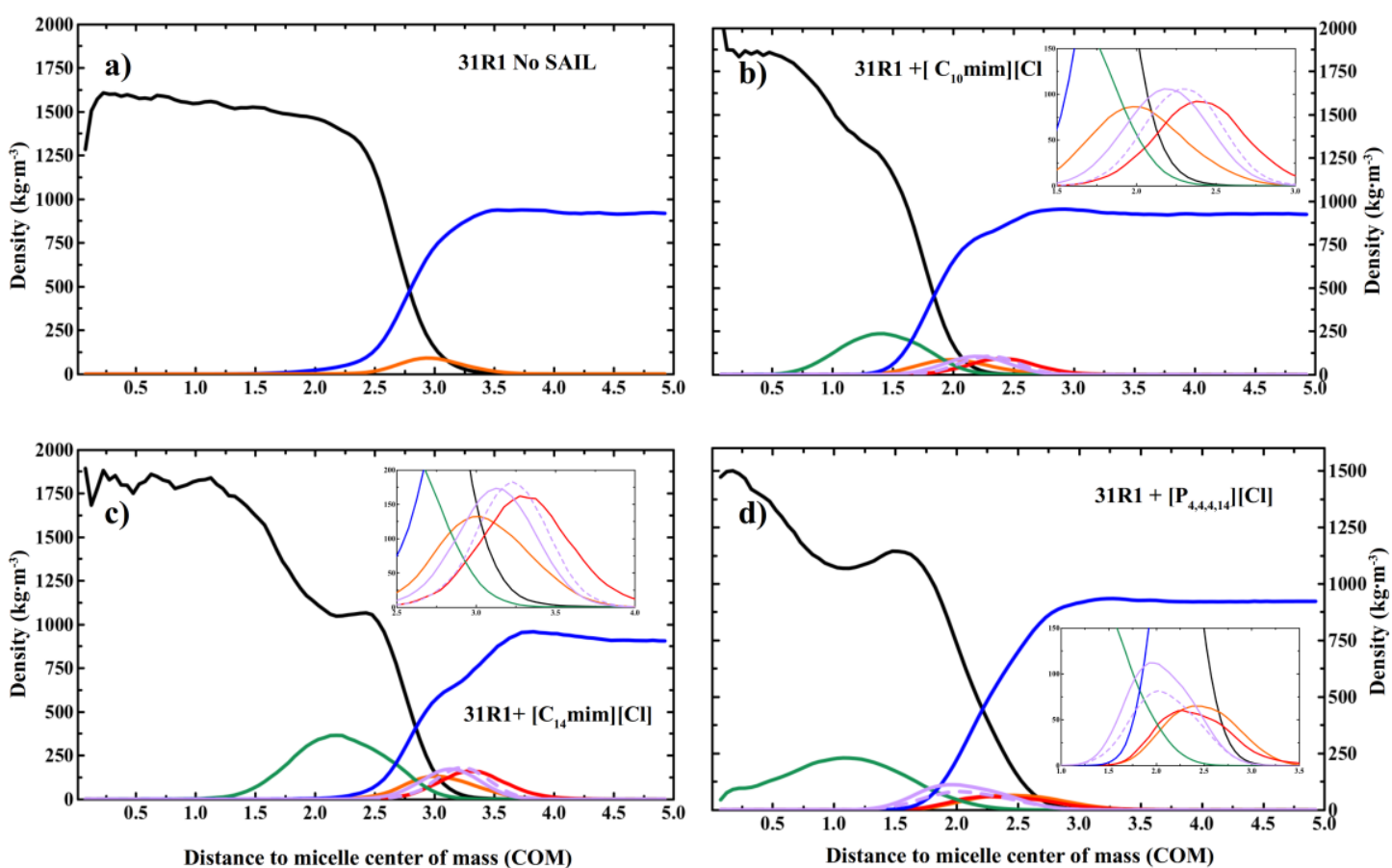

Figure S11. Micelle density profiles for 31R1 solutions without SAILs (a) and after addition of $\left[\mathrm{C}_{10} \mathrm{mim}\right] \mathrm{Cl}$ (b), $\left[\mathrm{C}_{14} \mathrm{mim}\right] \mathrm{Cl}(\mathrm{c})$ and $\left[\mathrm{P}_{4,4,4,14}\right] \mathrm{Cl}(\mathrm{d})$. The colour code is the same as in Figure S9. The insets show a blow up of the head-group region for the systems with SAIL. 


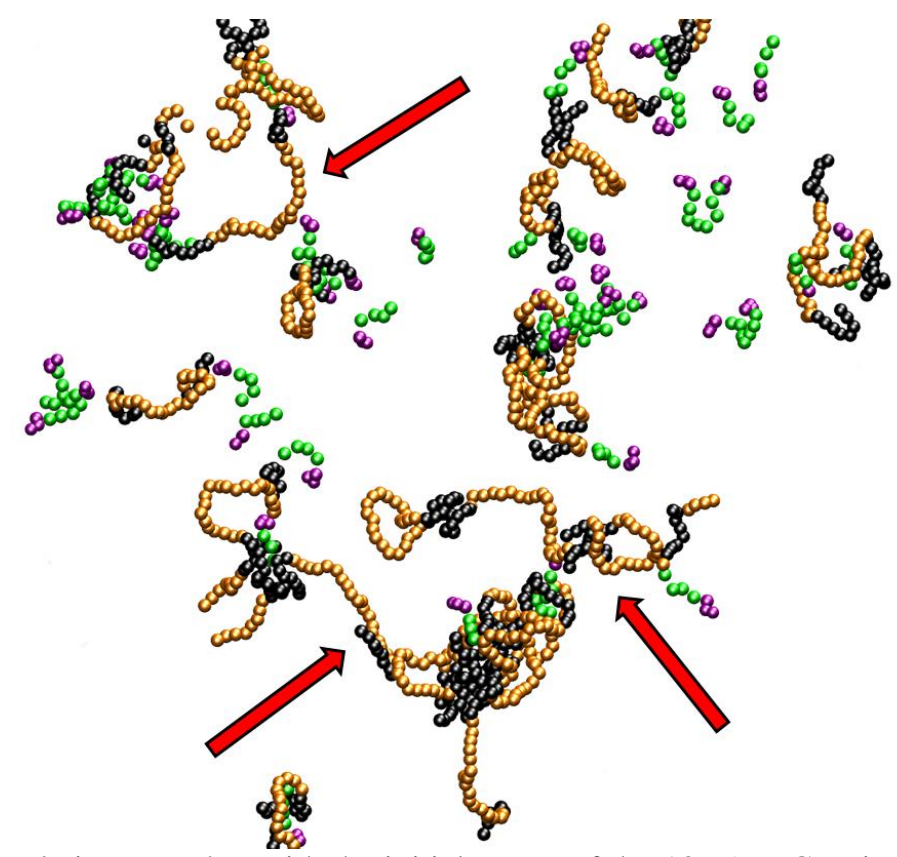

Figure S12. MD simulation snapshot with the initial stages of the $10 \mathrm{R} 5+\left[\mathrm{C}_{14} \mathrm{mim}\right] \mathrm{Cl}$ micelle formation at the cloud point temperature. Red arrows highlight the cross-linking between small aggregates. 